\title{
Cyanidin-3-o-glucoside directly binds to ERo36 and inhibits EGFR-positive triple-negative breast cancer
}

\author{
Li Wang ${ }^{1, *}$, Haifeng Li ${ }^{1, *}$, Shiping Yang ${ }^{1}$, Wenqiang Ma ${ }^{1}$, Mei Liu ${ }^{2}$, Shichao Guo ${ }^{1}$, \\ Jun Zhan ${ }^{3}$, Hongquan Zhang ${ }^{3}$, Suk Ying Tsang ${ }^{4}$, Ziding Zhang ${ }^{1}$, Zhaoyi Wang ${ }^{5}$, \\ Xiru Lí ${ }^{2}$, Yang-Dong Guo ${ }^{1}$, Xiangdong $\mathbf{L i}^{1}$ \\ ${ }^{1}$ State Key Laboratory of the Agro-Biotechnology, College of Horticultural Science, China Agricultural University, Beijing, \\ China \\ ${ }^{2}$ Department of General Surgery, The 301th Hospital of PLA, Beijing, China \\ ${ }^{3}$ Key Laboratory of Carcinogenesis and Translational Research, Ministry of Education, State Key Laboratory of Natural and \\ Biomimetic Drugs, Peking University Health Science Center, Beijing, China \\ ${ }^{4}$ School of Life Sciences and State Key Laboratory of Agro-Biotechnology, Chinese University of Hong Kong, Hong Kong, \\ China \\ ${ }^{5}$ Beijing Shenogen Pharma Group, Beijing, China \\ *These authors have contributed equally to this work \\ Correspondence to: Yang-Dong Guo, email: yaguo@cau.edu.cn \\ Xiru Li, email: 2468li@ sina.com \\ Zhaoyi Wang, email: charlie.wang@shenogen.com \\ Xiangdong Li, email: xiangdongli@cau.edu.cn
}

Keywords: Cy-3-glu, ERa36, EGFR, triple-negative breast cancer, apoptosis

Received: December 03, $2015 \quad$ Accepted: September 02, $2016 \quad$ Published: September 15, 2016

\section{ABSTRACT}

Anthocyanins have been shown to inhibit the growth and metastatic potential of breast cancer (BC) cells. However, the effects of individual anthocyanins on triplenegative breast cancer (TNBC) have not yet been studied. In this study, we found that cyanidin-3-o-glucoside ( $\mathrm{Cy}-3$-glu) preferentially promotes the apoptosis of TNBC cells, which co-express the estrogen receptor alpha 36 (ERa36) and the epidermal growth factor receptor (EGFR). We demonstrated that $\mathrm{Cy}-3$-glu directly binds to the ligand-binding domain (LBD) of ERa36, inhibits EGFR/AKT signaling, and promotes EGFR degradation. We also confirmed the therapeutic efficacy of Cy-3-glu on TNBC in the xenograft mouse model. Our data indicates that $\mathrm{Cy}-3-\mathrm{glu}$ could be a novel preventive/therapeutic agent against the TNBC co-expressed ERa36/EGFR.

\section{INTRODUCTION}

Triple-negative breast cancer (TNBC), one of multiple clinical subtypes of breast cancer (BC) defined by a lack of expression of estrogen receptors (ER), progesterone receptors (PR), and the HER-2/neu epidermal growth factor receptors [1], accounts for 10$15 \%$ of all $\mathrm{BC}$ cases [2]. TNBC often responds poorly to available chemotherapies [3]. Due to the lack of preclinical biomarkers for this subtype of $\mathrm{BC}$, the suitable clinical strategies for its treatment and prevention are barely defined. Accordingly, the identification of specific and effective molecular targets for effective therapies in TNBC is an urgent and unmet need.

TNBC is sometimes classified into a "basal-type" cancer group, which is frequently defined by cytokeratin
5/6 and epidermal growth factor receptor (EGFR) positive staining. Approximately $75 \%$ of basal-type BCs are TNBC. The overexpression of EGFR has been demonstrated in up to $88.5-89.5 \%$ of TNBC patients [4, 5] since EGFR was identified as a therapeutic target for TNBC patients in 2011 [6]. Nevertheless, no clear criteria have been standardized yet [7].

Although TNBC is characterized by a lack of ER $\alpha$ expression, several studies showed that an ovariectomy did prevent the progression of TNBC. A case-control study of 187 TNBC cases described a 2.5 increased risk for women who used oral contraceptives (OCs) for more than one year compared to women who used OCs for less than one year or never [8]. Altogether, these data implied that estrogen still plays a critical role in the etiology of TNBC. The ER $\alpha 36$, a $36-\mathrm{kDa}$ variant of $\mathrm{ER} \alpha$, is highly 
expressed in TNBC [9] and has been shown to be involve in membrane-initiated and rapid estrogen signaling [10]. EGFR was found to have therapeutic effects on TNBC and is now undergoing preclinical/clinical investigations [11]. These studies strongly emphasized the causal link between ER $\alpha 36$ and the EGFR signaling pathway in the etiology of TNBC [9].

Anthocyanins belong to a group of molecules called flavonoids, are derived from anthocyanidins, contribute to the intense color of many fruits, vegetables, and pigments, and are abundant in our daily diet [12]. There are six particularly important anthocyanidins, including cyanidin, delphinidin, pelargonidin, malvidin, peonidin and petunidin. Due to their instabilities in nature, acylated anthocyanidins are most frequently produced and glycosylated 2 or 3-fold with monosaccharides [13] to form anthocyanins. Anti-oxidant [14], anti-inflammatory [15], and anti-proliferative $[16,17]$ activities for a mixture of anthocyanins have been reported. However, the antiTNBC effects of the individual anthocyanins have not been well studied. As Cy-3-glu is the most abundant anthocyanin pigment in many vegetables and fruits [18], the goal of this study was to figure out the mechanism mediating the effects of $\mathrm{Cy}-3$-glu in the prevention of TNBC.

\section{RESULTS}

\section{Cy-3-glu inhibits the growth of TNBC cells}

Cy-3-glu is the most widespread glycoside class of anthocyanin pigments in vegetables and fruits (Supplementary Table S1) [19]; the contents of Cy-3-glu is usually more than 10-100 times higher than delphinidin3-glucoside, petunidin-3-glucoside, peonidin-3-glucoside, pelargonidin-3-glucoside and malvidin-3-glucoside. To verify the efficacy of Cy-3-glu against BC, several cell lines representing different clinical subtypes of $\mathrm{BC}$ were examined. We treated MDA-MB-231 cells with low dose Cy-3-glu (5 and $10 \mu \mathrm{M})$ for a longer time (7 d) and found that the growth of MDA-MB-231 cells was significantly inhibited $(\mathrm{p}=0.022)$ at $7 \mathrm{~d}$ (Supplementary Figure S1A). In order to study the mechanism and shorten the experiment time, we use the higher doses of Cy-3-glu (150 and $500 \mu \mathrm{M}$ ) and set up the time points at 24 and 48 $\mathrm{h}$, respectively. We observed no significantly additional cytotoxicity at $48 \mathrm{~h}$ with higher dose $(150$ and $500 \mu \mathrm{M})$ of Cy-3-glu treatment (Supplementary Figure S1B). Treatment with both doses, the 150 and $500 \mu \mathrm{M}$ of Cy-3glu, significantly inhibited TNBC cell growth, as shown in Figure 1. Specifically, MDA-MB-231 ( $<0.001$ at $48 \mathrm{~h})$, MDA-MB-436 (p $<0.001$ at $24 \mathrm{~h}$ and $48 \mathrm{~h} ; 500 \mu \mathrm{M})$ and BT20 cells ( $<<0.5$ at $24 \mathrm{~h}$ and $\mathrm{p}<0.001$ at $48 \mathrm{~h} ; 500 \mu \mathrm{M})$. Actually, a greater growth inhibitory effect was observed on MDA-MB-231 cells (Figure 1A and 1B), and this may due to different sensitivities of individual cell lines.
In comparison, no significant changes were obtained in the non-TNBC cells, including the MCF-7, SK-BR-3 and the non-cancerous breast epithelial cells MCF-10A treated with $500 \mu \mathrm{M}$ Cy-3-glu for $24 \mathrm{~h}$ and $48 \mathrm{~h}$ (Figure 1A and 1B). Morphological analysis of the cells also showed a significant change in the MDA-MB-231, MDA-MB-436 and BT20 cells, which were characterized by decreased cell volume, concomitant shrinking, pyknotic nucleus and foaming of the cell membrane. In comparison, the MCF7, SK-BR-3 and MCF-10A cell lines did not show any of the characteristic features observed in the TNBC after the $48 \mathrm{~h}$ treatment (Figure 1C). Taken together, the data demonstrated that $\mathrm{Cy}-3$-glu inhibits TNBC cell growth more effectively compared to non-TNBC cells, and has no effect on non-cancerous breast epithelial cells.

\section{Cy-3-glu induces TNBC MDA-MB-231 cell apoptosis with no effect on the cell-cycle}

We next asked whether the suppression of cell growth by Cy-3-glu was due to any effect on the cellcycle. To evaluate the effects of Cy-3-glu on the cell cycle, flow cytometry analysis was performed on MDA-MB-231 cells. We observed no alterations in the sub-G0/G1 phase, $\mathrm{S}$ phase and $\mathrm{G} 2 / \mathrm{M}$ population after $\mathrm{Cy}-3$-glu treatment for 12, 24 and $48 \mathrm{~h}$ (Figure 2A and Supplementary Figure S2). We also found no significant changes in the mRNA expression of cell cycle regulatory molecules (cyclinB1, cyclinD1, cyclinE, CDK2 and CDK4) after Cy-3-glu treatment for $24 \mathrm{~h}$ (Figure 2B). In addition, we detected no changes in the proliferating cell nuclear antigen (PCNA) by Western blot analysis (Figure 2C). These results indicated that Cy-3-glu inhibited MDAMB-231 cell growth independent of cell-cycle arrest. However, quantitation of flow cytometric analysis results demonstrated that Cy-3-glu treatments (48, 96 and 192 h) induced a dramatic increase in the amount of apoptotic cells in the experiment with MDA-MB-231 cells along the time (Figure 2D). The TUNEL assay also showed an increased number of dead cells (Figure 2E) and positive cells from $5.04 \%$ to $13.20 \%$ by flow cytometry (Figure 2F) after Cy-3-glu treatment of MDA-MB-231 cells. In summary, these results indicated that $\mathrm{Cy}-3$-glu inhibited growth of MDA-MB-231 cells by inducing apoptotic death rather than cell-cycle arrest.

\section{Cy-3-glu induces TNBC MDA-MB-231 cell death by apoptosis, not necroptosis, by up caspases cascade pathway}

Since both apoptosis [20] and necroptosis [21] are considered two of the important mechanisms of $\mathrm{BC}$ cell death. We first evaluated RIP3 expression in MDAMB-231 cells, using the mouse pancreas as a positive control (Figure 3A). Our failure to detect RIP3 expression in MDA-MB-231 cells treated with Cy-3-glu, suggested 
that necroptosis was not involved in Cy-3-glu-induced cells death. Apoptosis mediated by the TNF cytokine is referred to as the extrinsic apoptosis pathway and is initiated by the binding of TNF to its cell surface receptors
(TNFRs) thus activating the initiator caspase-8, followed by activation of the downstream effector caspase-3, resulting in cleavage of critical cellular proteins, ultimately leading to cell death [22]. Indeed, we observed

A

MCF-10A

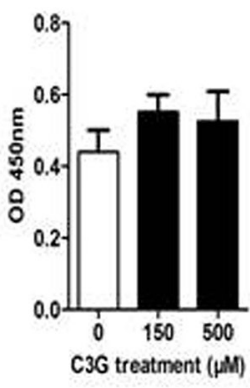

B

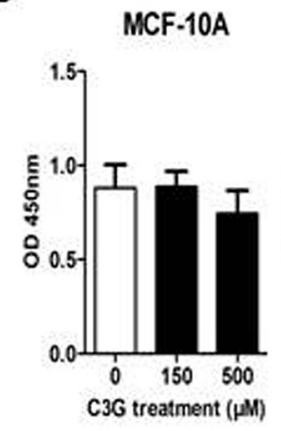

MCF-7

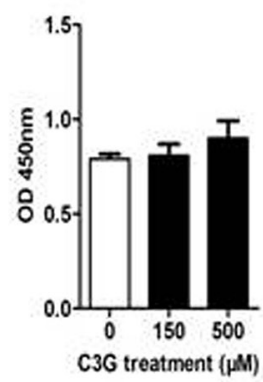

SK-BR-3

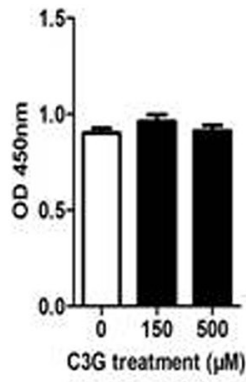

MDA-MB-231

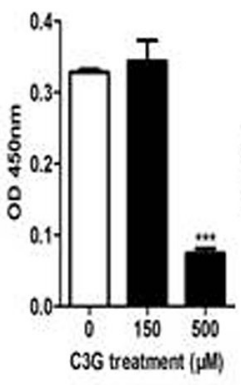

MDA-MB-436

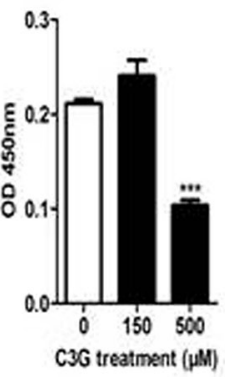

BT20

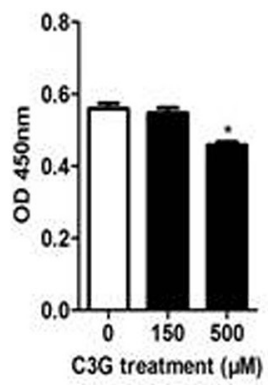

\section{$48 h$}
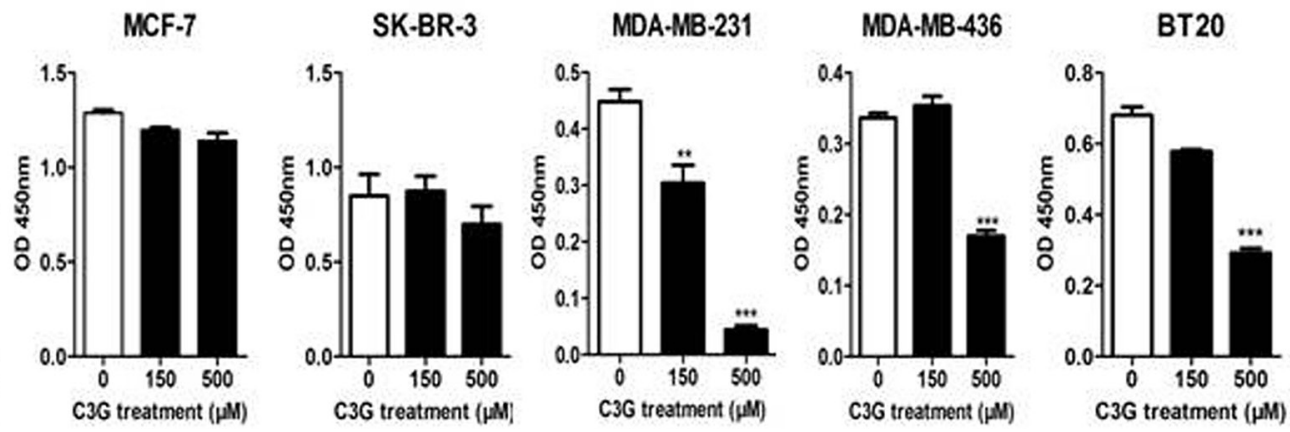

C

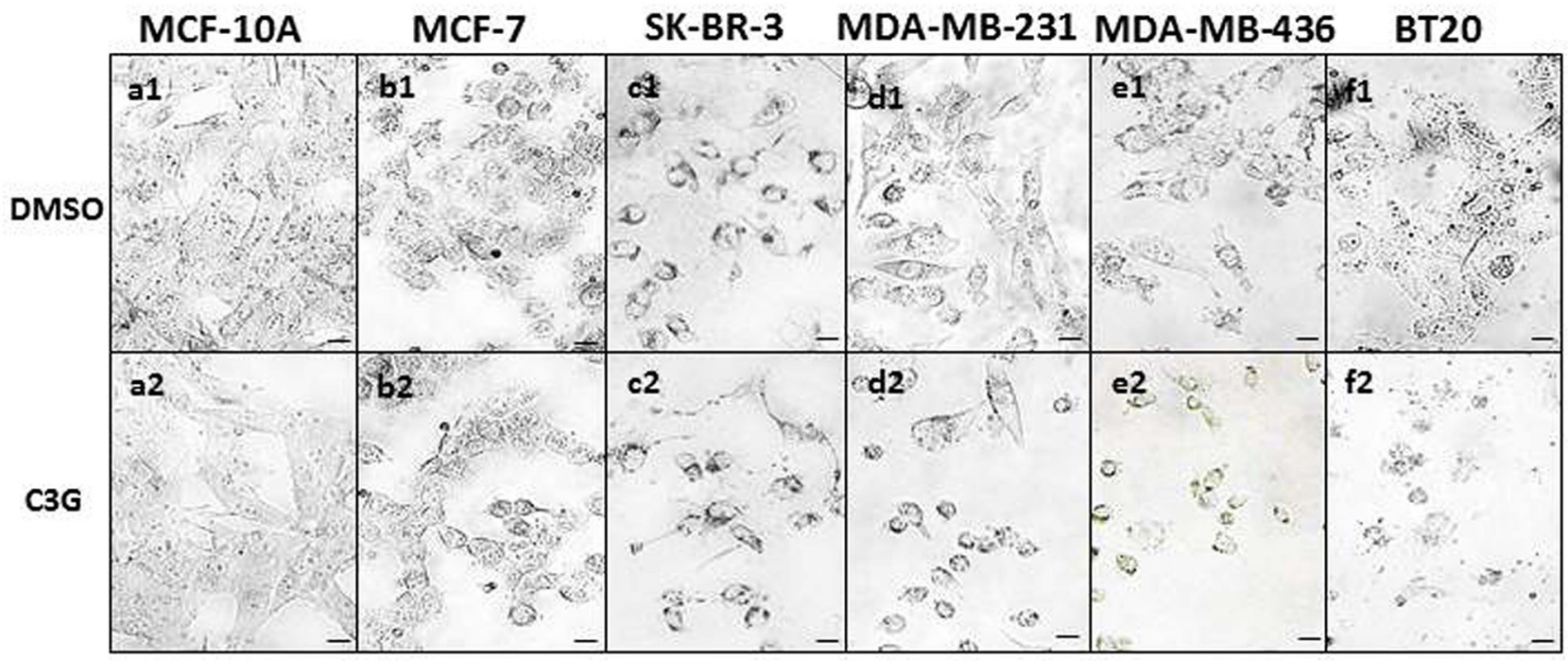

Figure 1: Cy-3-glu inhibits the growth of TNBC cells. A and B. the prolifereation of cells analyzed by the CCK-8 assay. Cells were treated with $150 \mu \mathrm{M}$ and $500 \mu \mathrm{M}$ Cy-3-glu for $24 \mathrm{~h}$ and $48 \mathrm{~h}$; DMSO serves as the vehicle control. C. cell morphology observed by phase-contrast microscopy corresponding to Figure 1B. The upper panel (a1 to f1) shows the controls, and the lower panel (a2 to f2) shows the Cy-3-glutreated cells after $48 \mathrm{~h}$. The results represent the mean \pm SEM from three independent experiments. Differences with $\mathrm{p}<0.05$ $(*), \mathrm{p}<0.01(* *)$ or $\mathrm{p}<0.001(* * *)$ were considered statistically significant. Scale bar is $100 \mu \mathrm{m}$. 
A

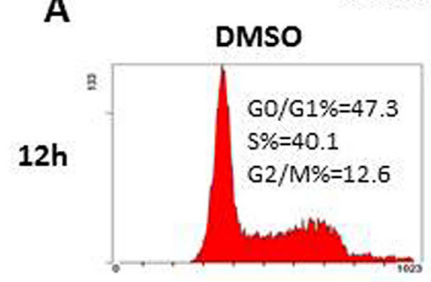

กับ

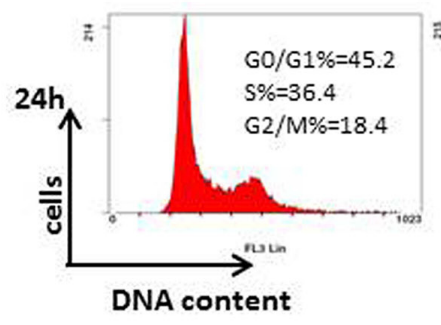

C
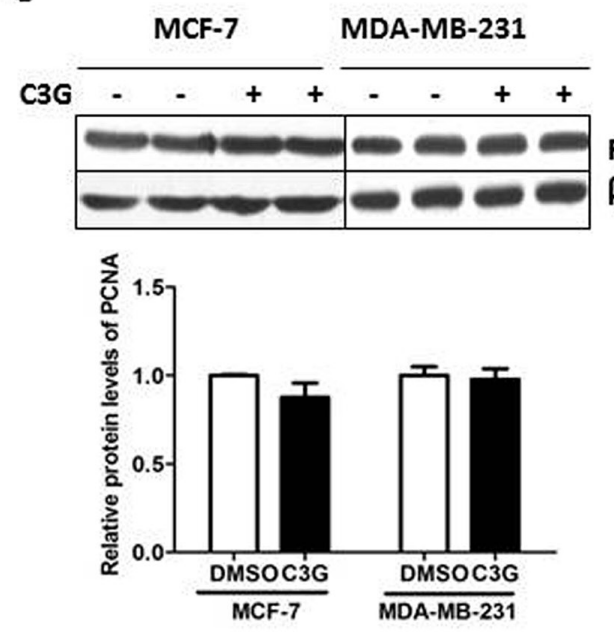

E

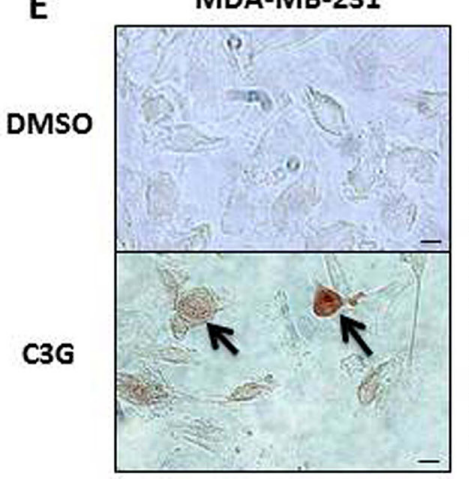

C3G

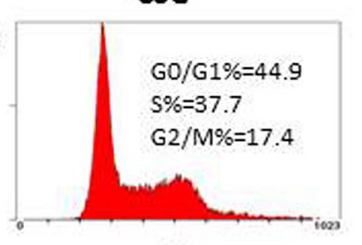

roum

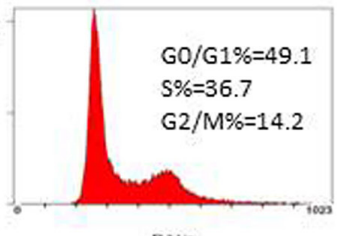

B

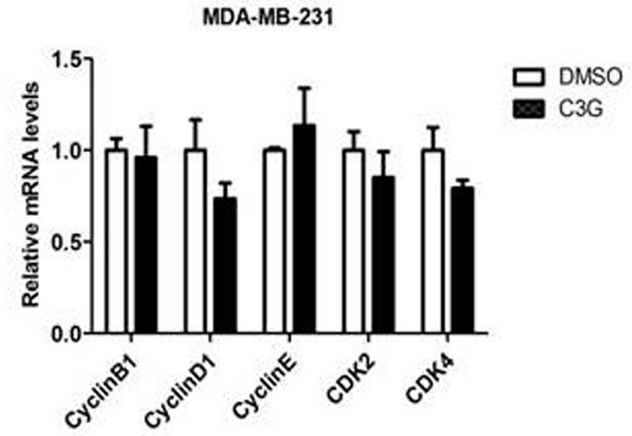

PCNA

D

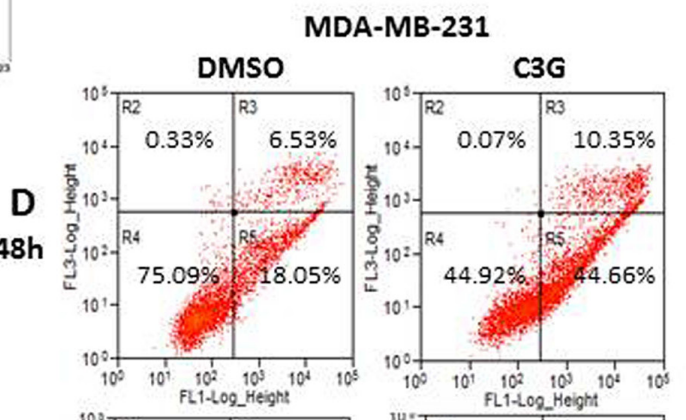

MDA-MB-231

$\beta$-actin
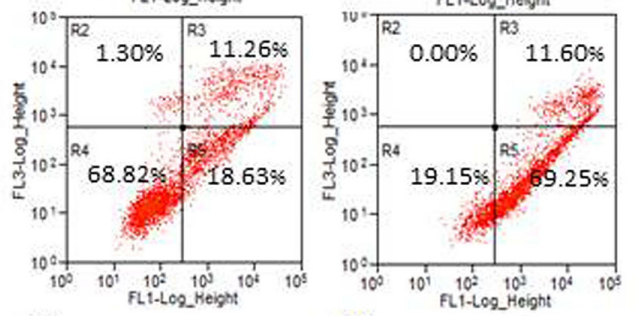

192h
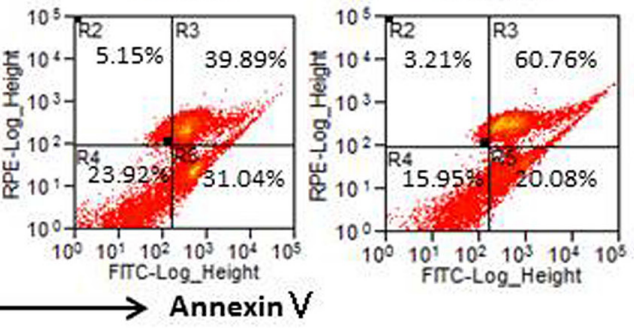

F
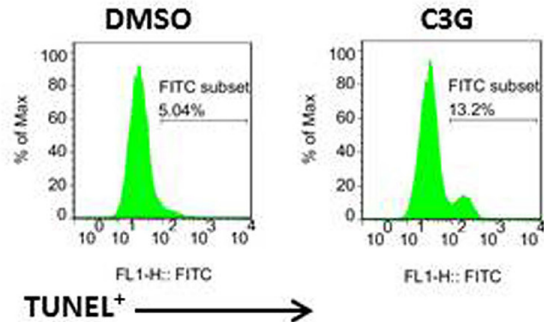

Figure 2: Cy-3-glu induces apoptosis in the MDA-MB-231 TNBC cells without affecting the cell-cycle. A. Evaluation of Cy-3-glu effects on MDA-MB-231 cell cycle progression by flow cytometry after $12 \mathrm{~h}$ and $24 \mathrm{~h}$ treatments. DNA content was measured by PI staining (x axis) and the population of cells was measured (y axis). B. relative mRNA expression levels of cyclin B1, cyclin D1, cyclin $E, C D K 2$ and $C D K 4$ after treatment with Cy-3-glu $(150 \mu \mathrm{M})$ for $24 \mathrm{~h}$ in MDA-MB-231 cells. C. upper panel, immunoblots of PCNA in MCF-7 and MDA-MB-231 cells untreated or treated with Cy-3-glu; $\beta$-actin was used as the internal control. Lower panel, bar graphs show the relative protein levels of PCNA. D. Annexin V/PI double staining for apoptotic MDA-MB-231 cells by flow cytometry at $48 \mathrm{~h}, 96 \mathrm{~h}$, and $192 \mathrm{~h} \mathrm{Cy-3-glu} \mathrm{treatment.} \mathrm{Phosphatidylserine} \mathrm{was} \mathrm{measured} \mathrm{by} \mathrm{annexin} \mathrm{V-FITC} \mathrm{staining} \mathrm{(x} \mathrm{axis),} \mathrm{and} \mathrm{apoptosis} \mathrm{was} \mathrm{measured} \mathrm{by} \mathrm{PI}$ staining (y axis). E. left panel, TUNEL staining to detect late apoptotic cells after Cy-3-glu treatment. Late apoptotic cells had brownish nuclear regions (arrows). Right panel, bar graphs show the number of TUNEL-positive cells. F. TUNEL staining for apoptotic MDAMB-231 cells after Cy-3-glu treatment for $48 \mathrm{~h}$ by flow cytometer, and the determined percentage of $\mathrm{TUNEL}^{+}$cells. The results represent the mean \pm SEM from three independent experiments. Scale bar is $100 \mu \mathrm{m}$. 
A

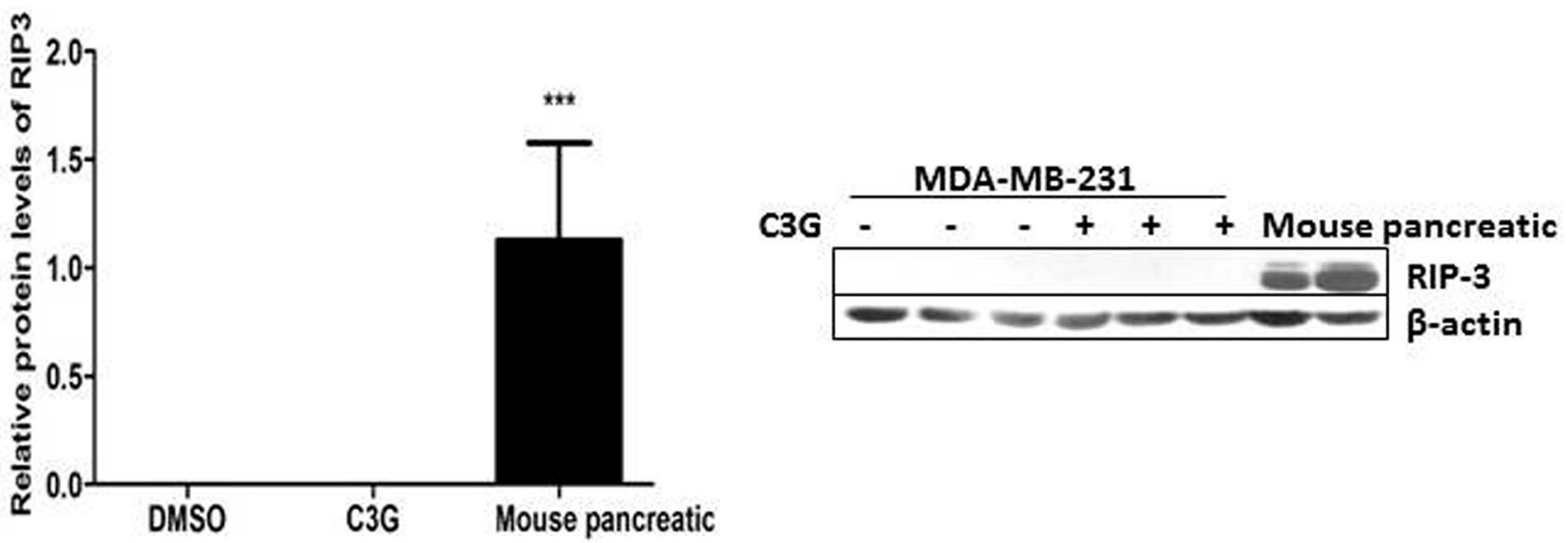

B
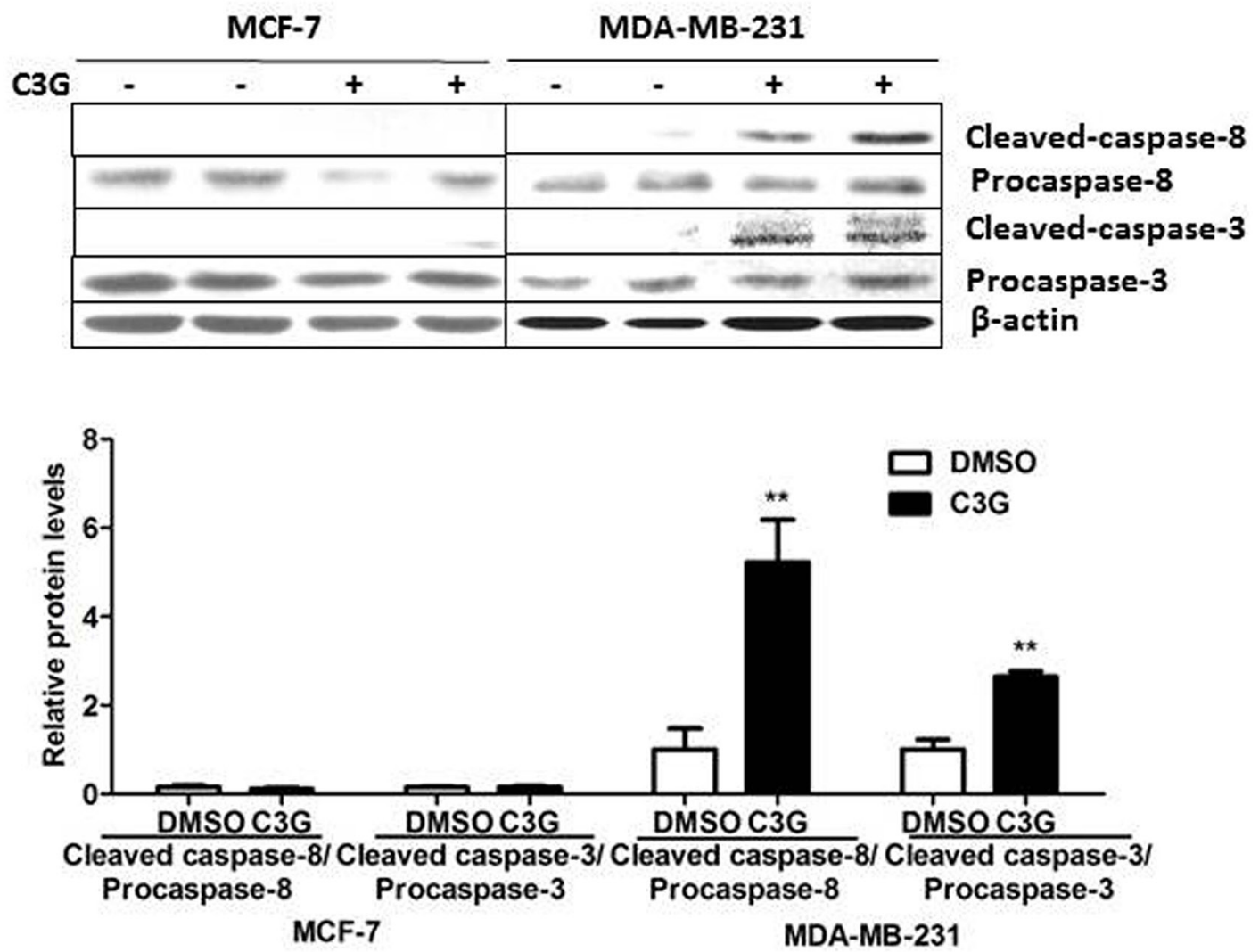

Figure 3: Cy-3-glu induces TNBC MDA-MB-231 cell death by the extrinsic apoptosis pathway, not necroptosis or by the intrinsic pathway. MCF-7 and MDA-MB-231 cells were treated with Cy-3-glu $(150 \mu \mathrm{M})$ for $24 \mathrm{~h}$ in FBS-free cell culture medium. A. left panel, bar graphs show the relative protein levels of RIP3. Right panel, RIP3 expression in MDA-MB-231 detected by Western blotting; the mouse pancreas was used as the positive control. B. upper panel, expression levels of cleaved-caspase-3 (17 kDa and $19 \mathrm{kDa})$, cleaved-caspase-8 $(47 \mathrm{kDa})$, caspase-3 $(35 \mathrm{kDa})$ and caspase-8 $(57 \mathrm{kDa})$. $\beta$-actin was the internal control. Lower panel shows the relative protein levels of cleaved-caspase-8 and cleaved-caspase-3 in MCF-7 and MDA-MB-231 cells. The results represent the mean \pm SEM from three independent experiments. Differences with $\mathrm{p}<0.05(*), \mathrm{p}<0.01(* *)$ or $\mathrm{p}<0.001(* * *)$ are considered statistically significant. 
an increase in cleaved-caspase- 8 after Cy-3-glu treatment, and consequently an increase of cleaved-caspase- 3 in MDA-MB-231 cells. On the other hand, no detectable cleaved-caspase- 8 or cleaved-caspase- 3 was observed in MCF-7 cells (Figure 3B). Together these results show that Cy-3-glu promoted cell apoptosis through activation of the caspases cascade in MDA-MB-231, but not in MCF-7 cells.

\section{Cy-3-glu induces TNBC MDA-MB-231 apoptosis via the non-mitochondrial pathway}

Superoxide dismutase (SOD), an important indicator of anti-oxidant capacity, reduces the superoxide anion to maintain the cellular redox homeostasis [23]. We determined the cellular SOD activity after Cy-3-glu treatment and found no significant changes in MDAMB-231 cells (Figure 4A, $p=0.530$ ). Mitochondria are a key player in apoptosis through the generation of reactive oxygen species (ROS) and release of cytochrome $\mathrm{C}$ and the subsequent activation of caspase- 9 and caspase-3/7 [24-26]. However, in our study we observed no significant mitochondrial function changes between MDA-MB-231 cells treated with DMSO or Cy-3-glu (Figure 4B). In addition, we failed to detect the expression of active caspase-9 (cleaved-caspase-9) in MDA-MB-231 cells (Figure 4C). These results indicated that $\mathrm{Cy}-3$-glu induces MDA-MB-231 cell apoptosis independent of the mitochondrial intrinsic pathway.

\section{Cy-3-glu counteracts estrogen-induced proliferation of TNBC cells}

Due to lack of ER $\alpha$ expression, a prevailing view is that estrogen signaling is not involved in the development and progression of ER-negative BC. However, consistent with a previous report [27], in the present study E2 induced phosphorylation of the AKT in MDA-MB-231 cells at different time periods. The results shown in Figure $5 \mathrm{~A}$ indicate that AKT phosphorylation occurred within 30 min after E2 application and sustained the activation for up to $120 \mathrm{~min}$. Additionally, we also found that Cy-3-glu decreased the level of p-AKT (Figure 5B) and inhibited estrogen-stimulated proliferation of MDA-MB-231 cells (Figure 5C). Actually, previous studies indicated that ER $\alpha 36$ is highly expressed in MDA-MB-231 cells, with lower levels found in ER-positive MCF-7 cells, and it is undetectable in the mammary epithelial cells MCF-10A $[9,10]$. We found that SNG162 significantly inhibited the growth of MDA-MB-231 cells stimulated by E2 as a positive control (Figure $5 \mathrm{C}$ ). In addition, the knocked down of ER $\alpha 36$ by shRNA in MDA-MB-231 cells (Figure 5D) revealed that the knocking down of ER $\alpha 36$ could significant counteract the growth inhibitory effect of $\mathrm{Cy}$ 3-glu on MDA-MB-231 cells (Figure 5E). From these results we inferred that $\mathrm{Cy}-3$-glu inhibits the growth of MDA-MB-231 cells, presumably through ER $\alpha 36$.

\section{Cy-3-glu binds to the LBD of ERa36 directly and inhibits its signaling pathway in TNBC cells}

To evaluate the binding affinity between Cy-3-glu and the LBD of ER $\alpha 36$, we first conducted molecular docking analysis using the Auto Dock program (www. scripps.edu/mb/olson/doc/autodock). The conformation is derived from co-crystallization data of the ER $\alpha 66$ conformation (Protein Data Bank Code: 2YJA). By docking the conformation of LBD-ER 236 with $\mathrm{Cy}-3$ glu (Figure 6B) and merging the conformations of LBDER $\alpha 36$ and LBD-ER $\alpha 66$ (Supplementary Figure S3A), we observed there are deletions of $\alpha 8$ and $\alpha 9$ helixes in ER $\alpha 36$ compare to ER $\alpha 66$, which lead to a more open binding pocket of ER $\alpha 36$. Molecular docking studies showed that Cy-3-glu had a stronger binding affinity score with ER $\alpha 36$ (binding energy is $-79.89 \mathrm{kcal} / \mathrm{mol}$ ) (Supplementary Figure S3B) than with ER $\alpha 66$ (binding energy is -28.27 $\mathrm{kcal} / \mathrm{mol}$ ) (Supplementary Figure S3C). Accordingly, compare to ERa66, Cy-3-glu was more stable and easier to fit into the ligand-binding pocket of ER $\alpha 36$. Furthermore, we checked the binding constant of Cy-3-glu to LBDER $\alpha 36$ and LBD-ER $\alpha 66$ by MST analysis, which allows for the sensitive detection of small-molecule binding to a protein target [28]. The data showed that Cy-3-glu binds to LBD-ER $\alpha 36$ with a KD of $31.4 \pm 1.88 \mu \mathrm{M}$ (Figure 6C) and almost no binding to LBD of ER $\alpha 66$ were observed compared to E2 positive control (Supplementary Figure $\mathrm{S} 4 \mathrm{~A}$ and $\mathrm{S} 4 \mathrm{~B}$ ). We have previously reported that EGFR is one of the most important downstream targets of activated ER $\alpha 36$ signaling [9]. Thus, to further determine the effects of Cy-3-glu on ERa36 signaling pathway, we analyzed the levels of EGFR and AKT phosphorylation. The analysis revealed that Cy-3-glu significantly inhibited the E2induced phosphorylation levels of the EGFR and AKT in TNBC MDA-MB-231, MDA-MB-436 and BT20 cells (Figure 6D). In summary, our data demonstrated that $\mathrm{Cy}-$ 3-glu directly binds to the ER $\alpha 36$ receptor and, in turn, inhibits its downstream signaling.

\section{The binding of Cy-3-glu to the LBD of ER 236 leads to the degradation of EGFR through the proteasome system in TNBC cells}

In order to further explore the different response between MDA-MB-231 and MCF-7 cells to Cy-3-glu, we mined the database (GEO34987) and screened out the top ten growth-related genes in MDA-MB-231 and MCF-7 cells (Table 1). Among them, we found that EGFR expression is 1.55 folds higher in MDA-MB-231 cells compared to MCF-7 cells. As the aberrant activation and over expression of EGFR leads to the dysregulation of signaling functions in cell proliferation, survival and cancer progression [29]. In addition, we additionally evaluated the expression profiles of ER $\alpha 36$ and EGFR in several BC cell lines from different clinical subtypes. We found a correlation between the expression of ER $\alpha 36$ 
and EGFR in the TNBC cell lines MDA-MB-231, MDA-MB-436 and BT20. In comparison, MCF-7 cells had lower level of ER $\alpha 36$ and nearly no expression of EGFR. Interestingly, SK-BR-3 cells express both ER $\alpha 36$ and EGFR but were insensitive to Cy-3-glu (Figure 1A, Figure $1 \mathrm{~B}$ and Figure $7 \mathrm{~A}$ ). This could be explained by the over expression of HER-2 that is involved in some counteractive mechanisms in SK-BR-3 cells. Furthermore, we found that $\mathrm{Cy}$-3-glu treatment dramatically decreased EGFR protein expression in MDA-MB-231 cells in a time- dependent manner (Figure 7B). However, no significant inhibition of EGFR expression at the mRNA level was detected after Cy-3-glu treatment (Figure 7C). It is well known that the intracellular degradation of proteins always takes place in the proteasome [30, 31]. As expected, the proteasome inhibitor MG132 completely restored the EGFR protein expression level (Figure 7D). Thus, promotion of EGFR degradation through the proteasome system was involved in the inhibition of TNBC cells growth mediated by Cy-3-glu.
A

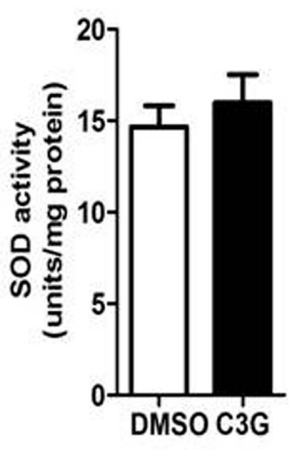

B

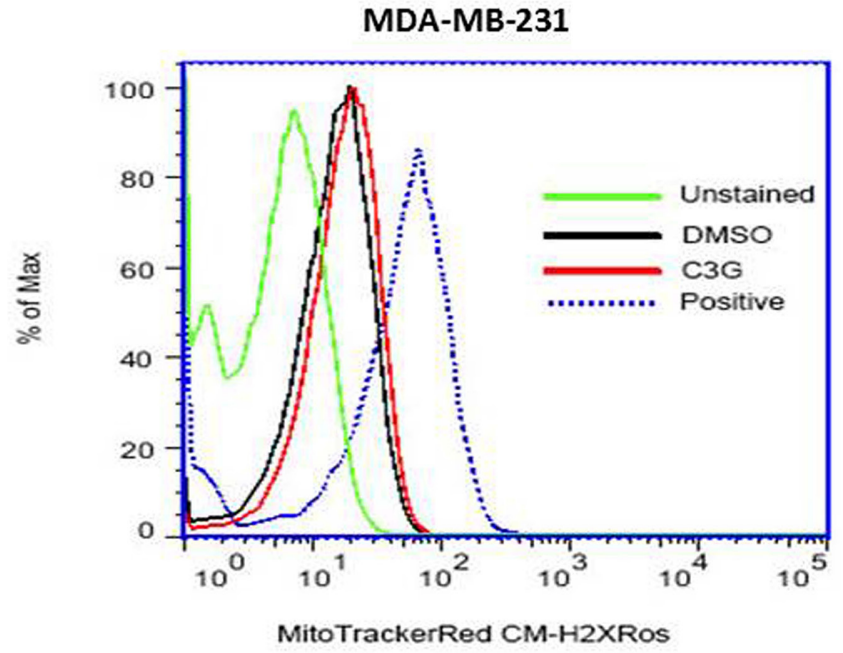

MitoTrackerRed CM-H2XRos

C

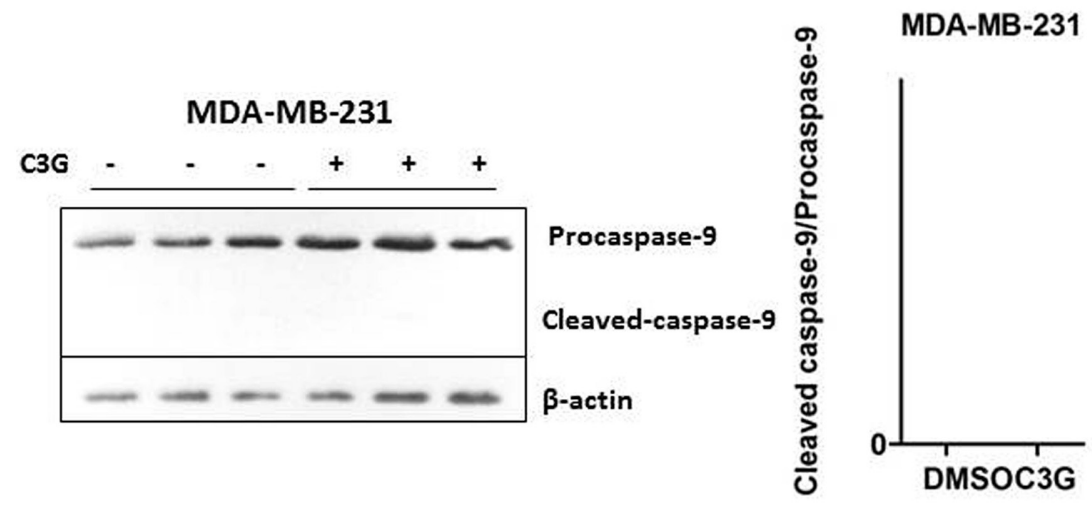

Figure 4: Cy-3-glu induces apoptosis of the MDA-MB-231 TNBC cells via the non-mitochondrial (extrinsic) pathway. Cells were treated with Cy-3-glu $(500 \mu \mathrm{M})$ for $24 \mathrm{~h}$. A. assessment of SOD activity between the DMSO and Cy-3-glu-treated groups. B. $30 \mathrm{~min}$ after the addition of CM-H2XRos to MDA-MB-231 cells, mitochondrial ROS fluorescent images were captured by flow cytometry. Mitochondrial ROS in MDA-MB-231 cells was stained by MitoTrackerRed CM-H2XRos. Non-stained groups were unstained and positive groups were treated with $\mathrm{H}_{2} \mathrm{O}_{2}$ before staining by MitoTrackerRed CM-H2XRos. X axis is the CM-H2XRos staining and y axis indicates the percentage of MDA-MB-231 cell populations between DMSO and Cy-3-glu treatments. C. left panel, the expression of caspase- 9 and cleaved-caspase-9 in MDA-MB-231 cells was detected by Western blotting. $\beta$-actin was used as the internal control. Right panel, bar graph shows the relative protein levels of cleaved-caspase-9/caspase-9. Data are represented as the mean \pm SEM $(n=3)$. Triplicate measurements were performed for each experiment. 
A

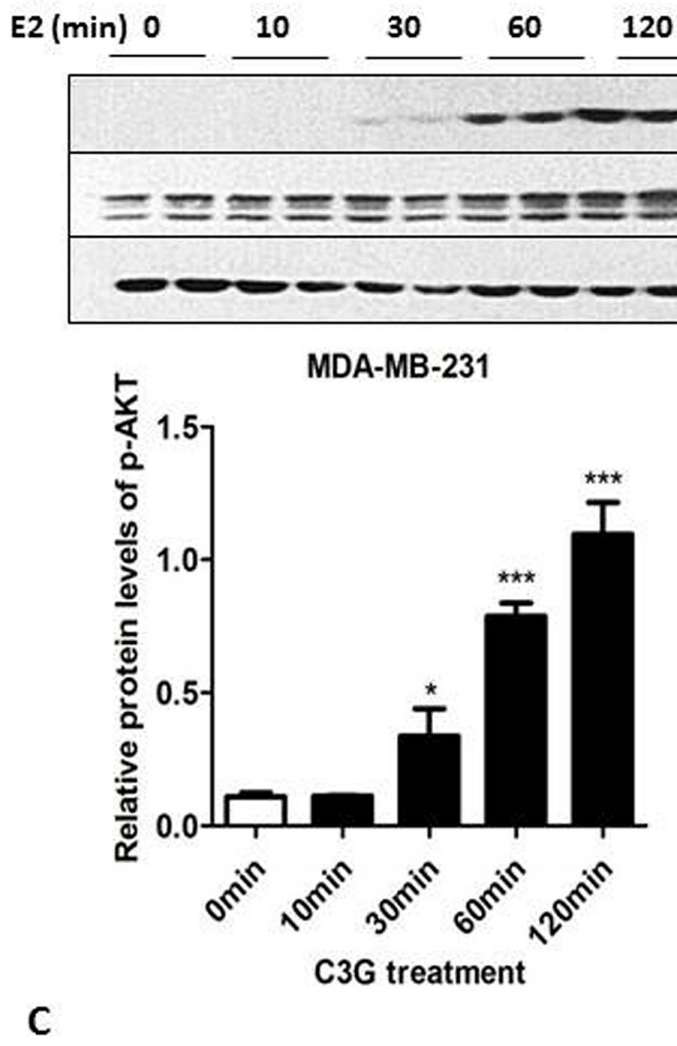

MDA-MB-231

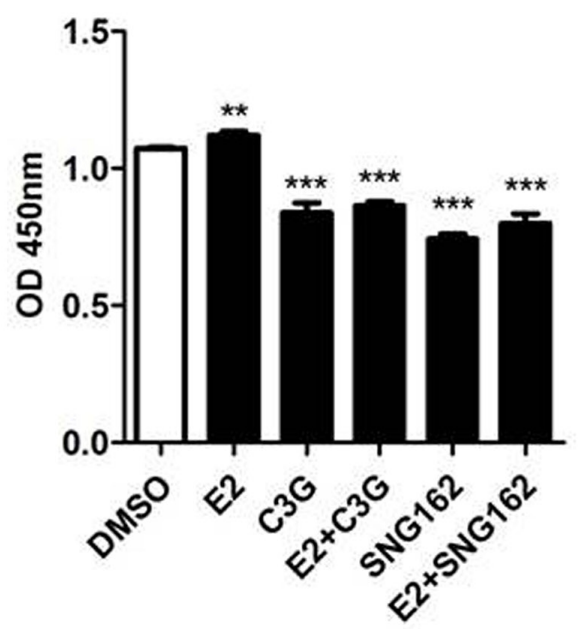

B
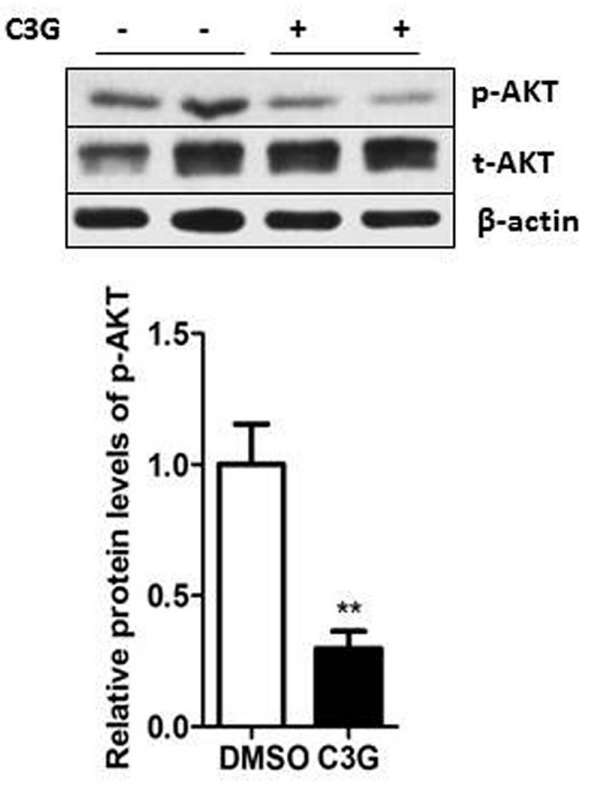

D

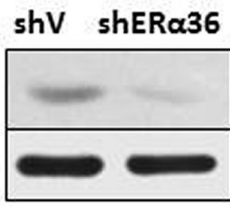

ERa36

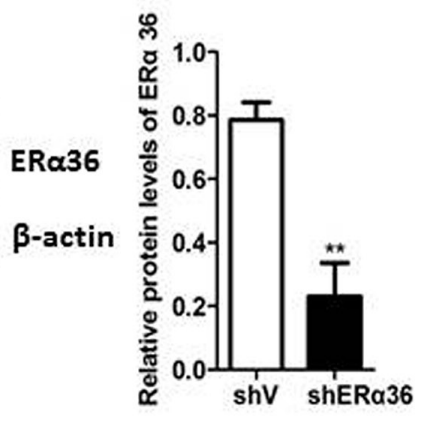

$\mathbf{E}$

MDA-MB-231

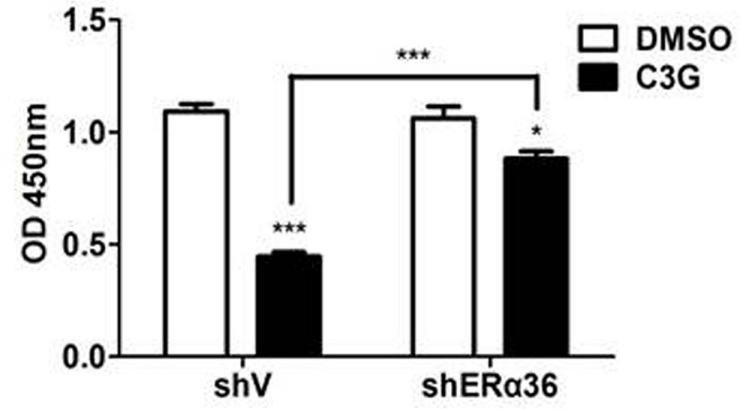

Figure 5: Cy-3-glu counteracts the estrogen-induced proliferation of MDA-MB-231 TNBC cells. A. MDA-MB-231 cells were treated with E2 $(100 \mathrm{nM})$ for the indicated time periods in FBS-free cell culture medium. The intensity of each band was quantified, and expressed as a percentage using the time 0 as reference. Upper panel, the expression of phosphorylated-AKT (p-AKT) and total AKT (t-AKT) were determined after E2 treatment. Lower panel, bar graphs show the relative protein levels of p-AKT/t-AKT. B. upper panel, the expression of t-AKT and p-AKT after Cy-3-glu $(150 \mu \mathrm{M})$ treatment was determined. $\beta$-actin was used as the internal control. Lower panel shows the ratios of p-AKT/t-AKT. C. MDA-MB-231 cells proliferation was analyzed by the CCK-8 assay at $24 \mathrm{~h}$. Cells were treated with $1 \mathrm{nM} \mathrm{E2}$ and $500 \mu \mathrm{M} \mathrm{Cy}-3$-glu, or E2 plus Cy-3-glu, or ERa36 inhibitor SNG162 or E2 plus ERa36 inhibitor SNG162, DMSO serves as the vehicle control. D. left panel, Western blot analysis of MDA-MB-231 cells transfected with a control vector (shV) and with the ER-a36 shRNA vector (shER $\alpha 36$ ). Right panel, bar graphs show the relative protein levels of ERa36. E. the proliferation of Cy-3-gluinduced MDA-MB-231 cells was analyzed after knock down of ER $\alpha 36$ by the shRNA method. The immunoblots shown here are from a representative experiment repeated three times with similar results. Differences with $\mathrm{p}<0.05(*), \mathrm{p}<0.01\left({ }^{* *}\right)$ or $\mathrm{p}<0.001\left({ }^{* *}\right)$ are considered statistically significant. 

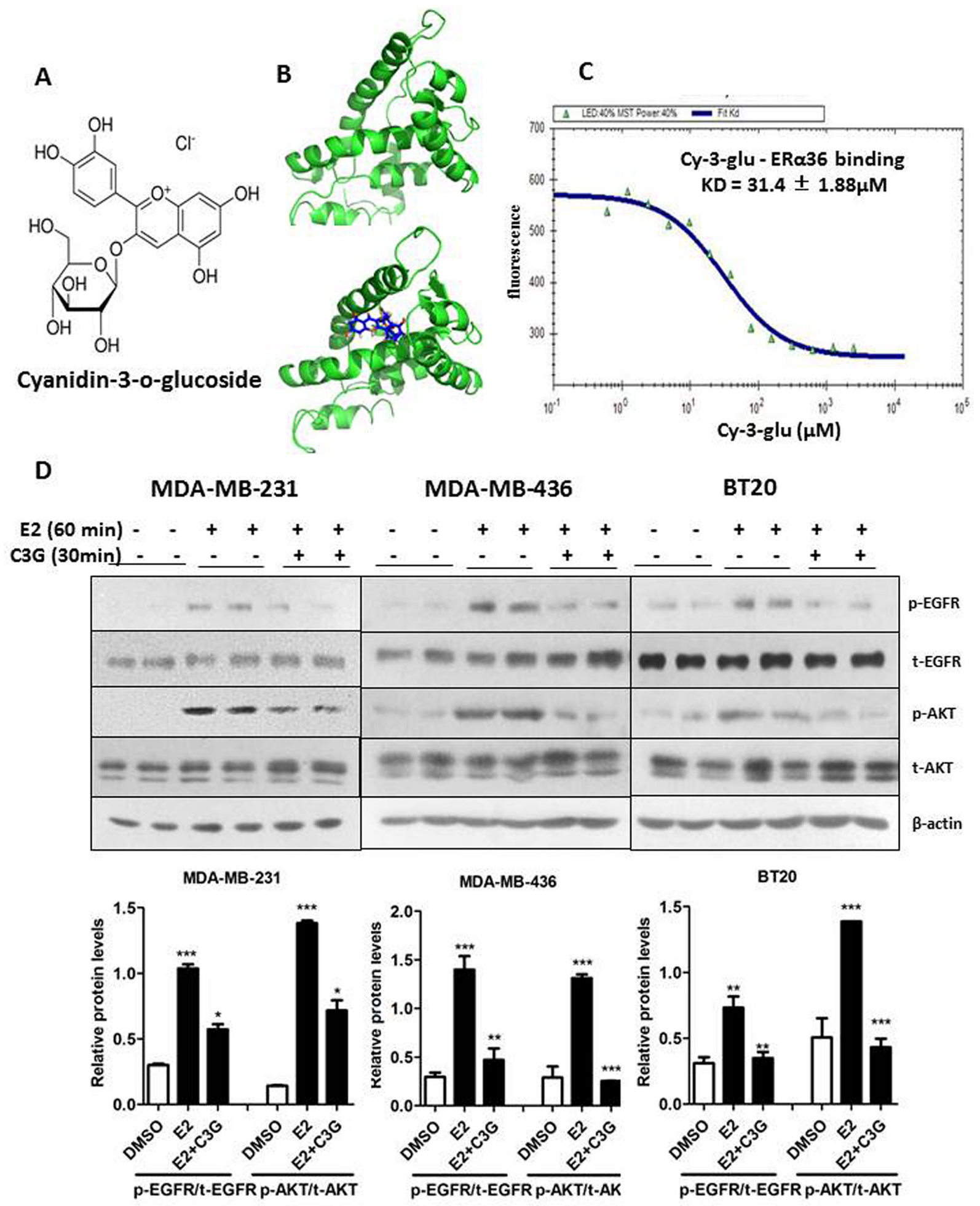

Figure 6: Cy-3-glu binds to LBD of ERa36 directly and inhibits its signaling pathway in TNBC cells. A. the chemical structure of Cy-3-glu. B. upper panel, a simulated docking model of the LBD-ERa36 (green color) complex. Lower panel, a simulated docking model of Cy-3-glu (dark blue color) in the pocket of LBD-ERa36 (green color). C. microscale thermophoresis analysis of Cy-3glu binding to LBD-ER $\alpha 36$. Purified LBD-ER 36 protein was first labeled with Cy5/Alexa 647 fluorescence dye. Cy-3-glu was titrated between 0.61 and $2500 \mu \mathrm{M}$ to the constant amount of labeled proteins $(100 \mathrm{nM})$ and the binding affinity is $31.4 \pm 1.88 \mu \mathrm{M}$. D. upper panel, phosphorylated-EGFR (p-EGFR), total EGFR (t-EGFR), p-AKT and t-AKT in TNBC cells were detected by Western blotting. Cy-3-glu $(150 \mu \mathrm{M})$ for $30 \mathrm{~min}$ before E2 $(100 \mathrm{nM})$ for $60 \mathrm{~min}$. MDA-MB-231, MDA-MB-436 and BT20 cells were harvested and lysed. Lower panel, bar graphs show the relative protein levels of p-EGFR/t-EGFR and p-AKT/t-AKT. The immunoblots shown here are from a representative experiment repeated three times with similar results. Differences with $\mathrm{p}<0.05(*), \mathrm{p}<0.01(* *)$ or $\mathrm{p}<0.001(* * *)$ are considered statistically significant. 
Table 1: Growth-related genes (top 10) expression analysis of MDA-MB-231 vs. MCF-7 mined from the NCBI database (GSE34987)

\begin{tabular}{lc}
\hline Gene name & Fold increase in MDA-MB-231 vs. MCF-7 (mean \pm SEM) \\
\hline GPR116 & $2.05 \pm 0.00$ \\
EMP1 & $1.92 \pm 2.00$ \\
ADORA2B & $1.64 \pm 1.00$ \\
PDGFC & $1.61 \pm 1.00$ \\
EGFR & $1.55 \pm 1.00$ \\
CSF1R & $1.54 \pm 0.00$ \\
IGF2BP3 & $1.49 \pm 0.00$ \\
CSF2 & $1.41 \pm 0.00$ \\
PKIB & $0.65 \pm 1.00$ \\
GREB1 & $0.60 \pm 0.00$ \\
\hline
\end{tabular}

GPR116: G protein-coupled receptor 116; EMP1: epithelial membrane protein 1; ADORA2B: adenosine A2b receptor; PDGFC: platelet derived growth factor C; EGFR: epidermal growth factor receptor; CSF1R: colony stimulating factor 1 receptor; IGF2BP3: insulin-like growth factor 2 mRNA binding protein 3; CSF2: colony stimulating factor 2 (granulocytemacrophage); PKIB: protein kinase (cAMP-dependent, catalytic) inhibitor beta; GREB1: growth regulation by estrogen in breast cancer 1 .

\section{Cy-3-glu inhibits the growth of MDA-MB-231 cells in vivo}

To examine the in vivo anticancer efficacy of Cy3 -glu, human breast tumor xenografts from athymic nude mice were used. By using a stably expressing luciferase (MDA-MB-231-luc) cell line to visualize and monitor the growth of $\mathrm{BC}$ in real-time, we observed that the tumor growth and volume in animals treated with Cy-3-glu were significantly smaller compared to the untreated control mice at the different time points evaluated (Figure 8A and 8B). The reduced tumor size and weight were further confirmed upon the scarification of mice (Figure $8 \mathrm{C}$ and $8 \mathrm{D}$ ). Histological analysis showed that $\mathrm{Cy}-3$-glu treatment significantly inhibited capillary formation (Figure 8E). The MVD was $25 \pm 1.66 / \mathrm{mm}^{2}$ (tumor group) vs. $4 \pm 0.33 / \mathrm{mm}^{2}$ (Cy3 -glu group). There were no differences in spleen and liver weights between the vehicle and $\mathrm{Cy}$-3-glu-treated groups, which further revealed that $\mathrm{Cy}$-3-glu has no toxicity (Supplementary Figure S5A and S5B). Body weights and food intake were monitored weekly as an indicator of overall health, and there were no differences between the control, tumor and $\mathrm{Cy}$-3-glu-treated groups (Supplementary Figure S5C and S5D). Consistent with the in vitro experiments, apoptosis (as indicated by cleaved-caspase-3 staining) of the tumor specimen was significantly increased in the $\mathrm{Cy}-3$-glu-treated group (Figure 8F). These results illustrated that $\mathrm{Cy}$-3-glu also inhibits TNBC growth in vivo.

\section{DISCUSSION}

The present study demonstrated that Cy-3-glu effectively promotes apoptotic cell death in a subset of TNBC cells that co-expressed ER $\alpha 36$ and EGFR. Mechanically, although ER 236 has not trans-membrane domain, it localizes to the caveolae of the plasma membrane invaginations. Cy-3-glu directly binds to the LBD of ERa36 and leads to the inhibition of its downstream EGFR/AKT signaling and promotes EGFR degradation through the proteasome system. This, in turn, drives the apoptosis of TNBC cells in a manner that is independent of the mitochondrial intrinsic pathway (Figure 9).

We observed the most significant inhibitory effect of Cy-3-glu on MDA-MB-231 cells among the three TN cell lines (MDA-MB-231, MDA-MB-436 and BT20). The expression of $\mathrm{p} 53$ was barely detectable in MDA-MB-436 cell line, whereas the overexpressed p53 were observed in MDA-MB-231 and BT20 cell lines. Moreover, as no expression of tumor suppressor protein RB1 in MDAMB-436 cell line, normal expression level in BT20 cell line, and overexpression in MDA-MB-231 cell line [32], the different responses from cell lines are mainly due to their different gene mutations and expressions.

It has previously been reported that $\mathrm{Cy}-3$-glu increases the proliferation of MDA-MB-231 by protecting the cells against the acrylamide-induced oxidative stress [14]. We observed the inhibitory effects of lower dose Cy-3-glu (5 and $10 \mu \mathrm{M}$ ) on MDA-MB-231 for 7 day and 
A
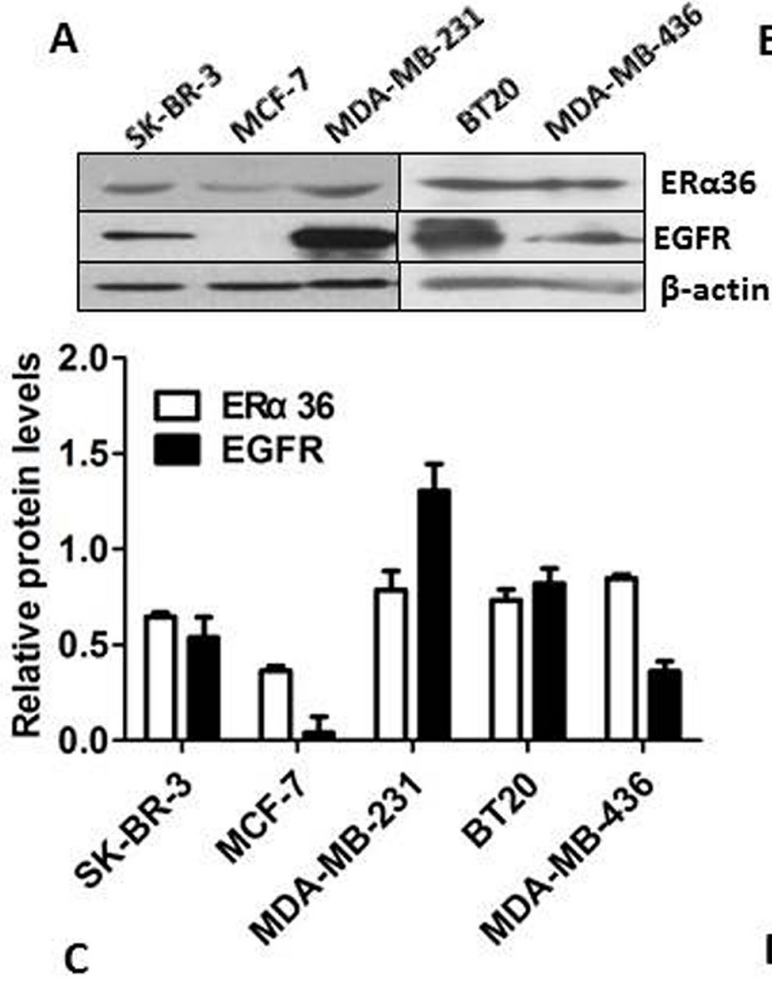

B
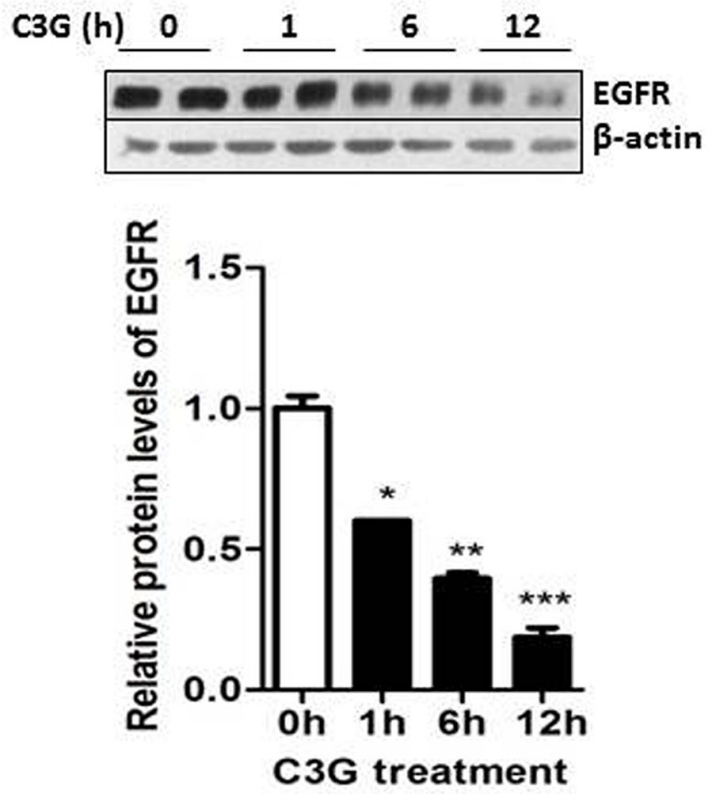

D
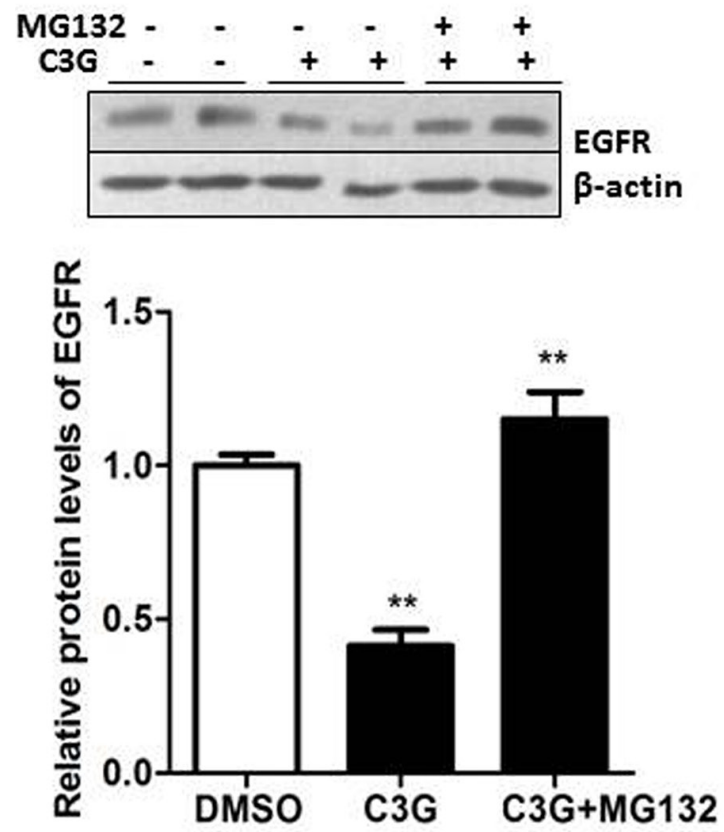

Figure 7: Binding of Cy-3-glu to the LBD of ERa36 leads to degradation of EGFR through the proteasome system in MDA-MB-231 TNBC cells. A. upper panel, the expression of ERa36 and EGFR in several BC cell lines. Lower panel, bar graphs show the relative protein levels of ER $\alpha 36$ and EGFR. B. MDA-MB-231 cells were treated with Cy-3-glu $(150 \mu \mathrm{M})$ for $0 \mathrm{~h}, 1 \mathrm{~h}, 6 \mathrm{~h}$ and 12 $\mathrm{h}$ in FBS-free cell medium. Upper panel, the expression of EGFR was determined after Cy-3-glu treatment. Lower panel, bar graphs show the relative protein levels of EGFR. C. upper panel, RT-PCR analysis of EGFR mRNA levels after Cy-3-glu $(150 \mu \mathrm{M})$ treatment at $0 \mathrm{~h}, 6$ $\mathrm{h}$ and $12 \mathrm{~h}$. Lower panel, bar graphs show the relative mRNA levels of EGFR. D. upper panel, EGFR degradation through the proteasome system was detected by Western blotting. MG132 was added 30 min before treatment with Cy-3-glu for $6 \mathrm{~h}$. The intensity of each band was quantified, and expressed as the percentage using the time $0 \mathrm{~h}$ as reference. Lower panel, bar graphs show the relative protein levels of EGFR. The immunoblots shown here are from a representative experiment repeated three times with similar results. Differences with $\mathrm{p}<$ $0.05(*), \mathrm{p}<0.01(* *)$ or $\mathrm{p}<0.001(* *)$ are considered statistically significant. 


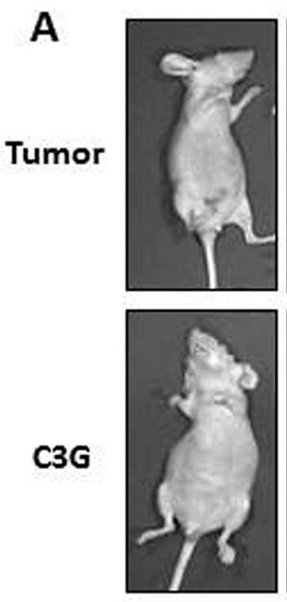

Baseline
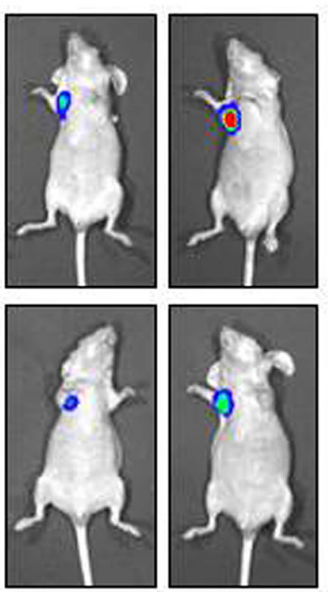

week 2

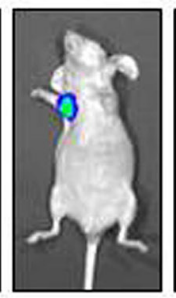

week 4
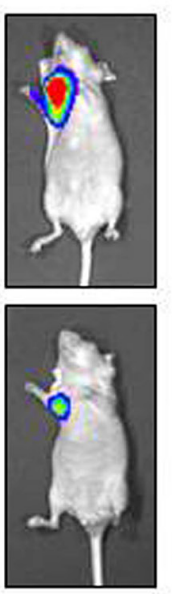

week 6
C

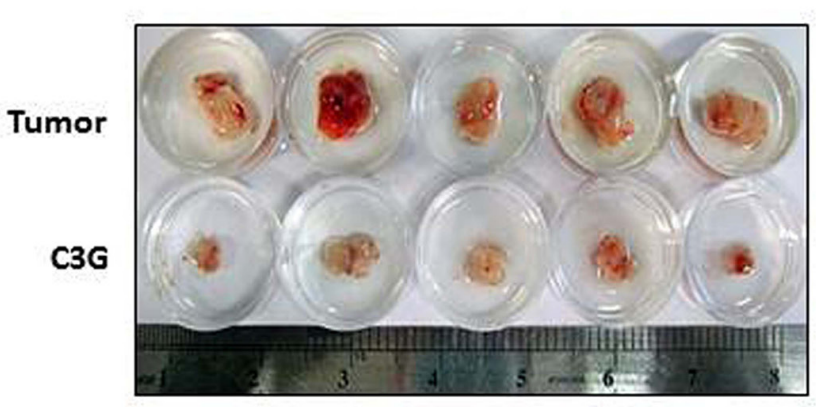

B

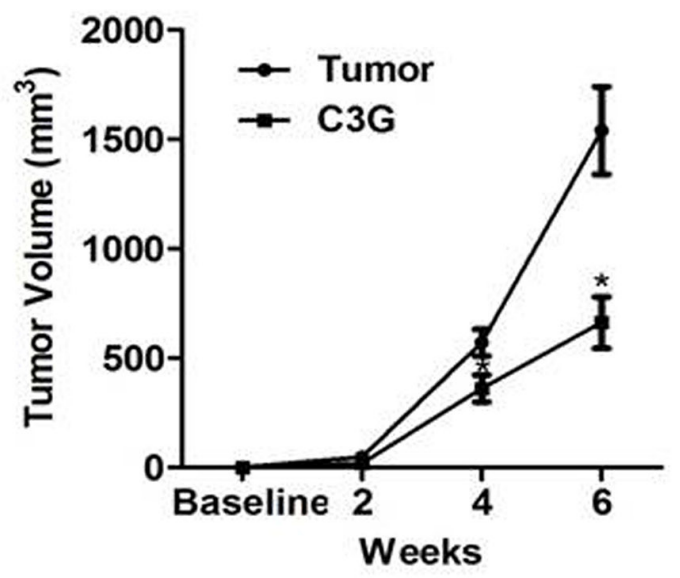

D

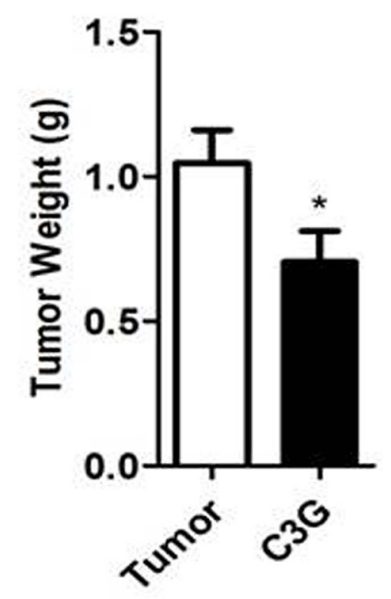

$\mathbf{E}$

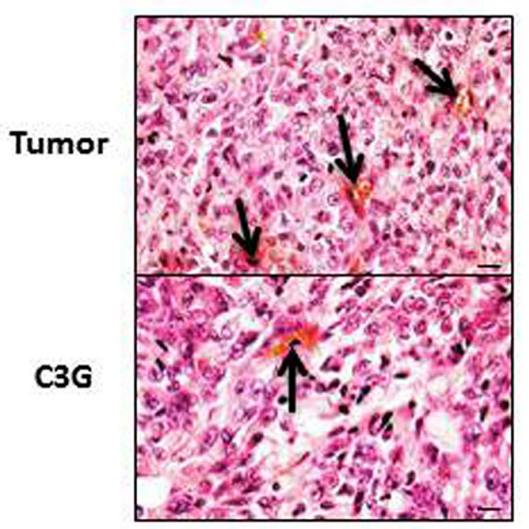

F

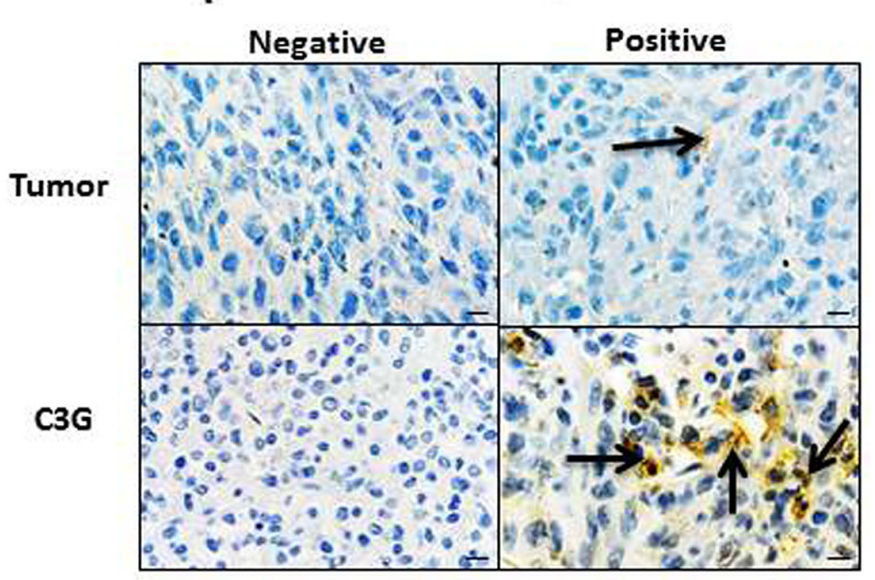

Figure 8: Cy-3-glu inhibits the growth of MDA-MB-231 cells in vivo. In vivo tumor growth monitored by Xenogen IVIS imaging at different time points after MDA-MB-231-luc tumor implantation in female BALB/c athymic mice $(\mathrm{n}=10)$ fed a control or Cy-3-glu diet for 6 weeks. A. orthotopic breast tumor growth was monitored in real time by bioluminescent imaging of luciferase activity in living mice using the cryogenically cooled IVIS-imaging system from baseline to week 6 post implantation. B. tumor growth was monitored (by a Vernier caliper) and presented as tumor volume in cubic millimeters, over a period of 6 weeks. C and D. photographic images of excised tumors were captured and its graphical representation of tumor weight $(\mathrm{n}=5)$. E. hematoxylin and eosin staining of tumor, and capillaries are red (arrows). F. cleaved-caspase-3 is brownish in the cytoplasm (arrows) of tumor and Cy-3-glu-treated mice tumors. Differences with $\mathrm{p}<0.05(*), \mathrm{p}<0.01(* *)$ or $\mathrm{p}<0.001(* * *)$ are considered statistically significant. Scale bar is $100 \mu \mathrm{m}$. 
higher doses Cy-3-glu (150 and $500 \mu \mathrm{M})$ at $24 \mathrm{~h}$ and $48 \mathrm{~h}$. The reason for this discrepancy may be the difference between the two studies in the treatment times of Cy-3-glu on MDA-MB-231 cells. They assessed the proliferation index at $4 \mathrm{~h}$ after the treatment, which is not a sufficiently long time for a cell cycle to occur. They also detected the inhibition of the production of ROS by Cy-3-glu after $4 \mathrm{~h}$, which must represent the acute phase of the cells response upon the stimulation rather than the stable effects of the Cy-3-glu. We also evaluated the anti-oxidant activities of Cy-3-glu, but the activities of SOD and the production of mitochondrial ROS exhibited no significant difference between treatment with Cy-3-glu and vehicle DMSO in MDA-MB-231 cells. Accordingly, our result indicates that the main function of Cy-3-glu is to induce MDA-MB-231 cells apoptosis rather than to act as an anti-oxidant. A deliberate study showed that whole blueberry extracts inhibited the growth and metastatic potential of MDAMB-231 cells $[33,34]$. However, the precise component in the mechanisms underlying the anti-TNBC activity remains to be defined. In this study, we determined that the anthocyanin, Cy-3-glu, is an effective agent against TNBC. The preventive and therapeutic efficacy of Cy-3- glu against TNBC needs to be further studied in a large clinical investigation.

Our results shown Cy-3-glu could bind to LBD of ER $\alpha 36$, and has no binding affinity to the LBDs of ER $\alpha 66$ and $\mathrm{ER} \alpha 46$. Compare to ER $\alpha 66$ and $\mathrm{ER} \alpha 46, \mathrm{ER} \alpha 36$ retains the partial LBD and possesses an extra, unique domain of 27 amino acids to replace the last 138 amino acids encoded by exons 7 and 8 . The truncated LBD of ER $\alpha 36$ suggests that it may have a spectrum of ligand selectivity different from ER $\alpha 66$ and ER 446 [35]. In addition, although $\mathrm{ER} \alpha 36$ has not trans-membrane domain, it localizes to the caveolae of the plasma membrane invaginations [36]. We and other groups have developed an affinity-purified rabbit polyclonal and monoclonal anti-ER $\alpha 36$ antibodies against 20 amino acids of the C-terminal of ER $\alpha 36[10,36]$, and demonstrated that ER $\alpha 36$ promoted non-genomic estrogen signaling and cell growth could be blocked by anti-ER $\alpha 36$ antibody [36-38]. All these results indicated that ER $\alpha 36$ can receive the extracellular molecule signal. Additionally, the glycoside moiety will confer a hydrophilic nature to $\mathrm{Cy}-$ 3-glu, but the other moiety of Cy-3-glu is an alkyl chain. Accordingly, it could be claimed that Cy-3-glu adsorbed to the cell membrane by the strength of electrostatic

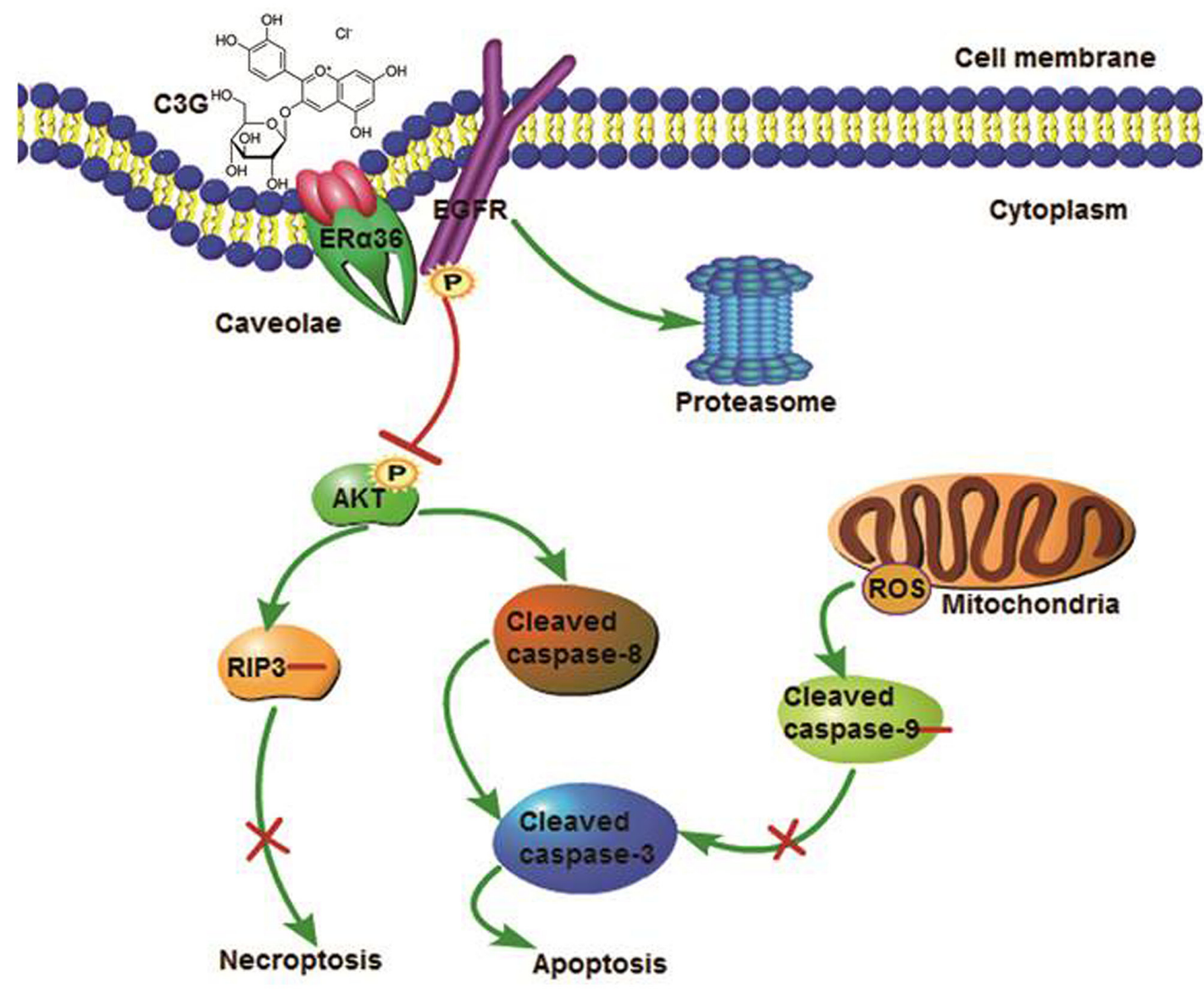

Figure 9: Putative mechanisms of the effects of $\mathbf{C y}$-3-glu action in the prevention of TNBC. Cy-3-glu binds directly to the LBD of ER 36 and then inhibits its downstream EGFR/AKT signaling and promotes EGFR degradation through the proteasome system, which in turn drives TNBC cells apoptosis, independent of the mitochondrial intrinsic pathway. 
Table 2: The sequences of the oligonucleotide primers and amplified products of real-time PCR

\begin{tabular}{lcc}
\hline Gene name & Sequence of primer (5'-3') & Amplified product (bp) \\
\hline GAPDH & F: ATGGGGAAGGTGAAGGTCG & 107 \\
R: GGGGTCATTGATGGCAACAATA & 105 \\
Cyclin D1 & F: ACCTGAGGAGCCCCAACAA & 156 \\
R: TCTGCTCCTGGCAGGCC & \\
Cyclin B1 & F: TGGACTATGACATGGTGCACTT & 110 \\
Ryclin E & F: GCCAGGTGCTGCATAACTG & \\
& R: TCTTCCTGGAGCGAGCCG & 134 \\
CDK2 & F: CCCTTTCTTCCAGGATGTGA & \\
& R: TGAGTCCAAATAGCCCAAGG & 78 \\
CDK4 & F: GAAACTCTGAAGCCGACCAG & \\
& R: ACATCTCGAGGCCAGTCATC & 148 \\
CDK6 & F: TCCCTCCTTTGAAGTGGATG & \\
& R: GTCACCTGGGGCTAAATGAA & 176 \\
EGFR & F: AGGCACGAGTAACAAGCTCAC & \\
\hline
\end{tabular}

F: forward; R: reverse; GAPDH: glyceraldehyde 3-phosphate dehydrogenase; CDK2: cyclin-dependent kinase 2; CDK4: cyclin-dependent kinase 4; CDK6: cyclin-dependent kinase 6; EGFR: epidermal growth factor receptor.

interactions of the polar groups of phospholipids and hydrophobic interactions with their alkyl chains.

Programed cell death has been reported to link three major routes, including apoptosis, necroptosis and autophagy [39, 40]. In particular, apoptosis and necroptosis are the two major modes of $\mathrm{BC}$ cell death [41, 42]. The determination for cells to undergo necroptosis or not is mediated by the factor RIP3 [43]. Since many cells do not express RIP3 [44], in this study we demonstrated that RIP3 was hardly detected in MDA-MB-231 cells. Thus, the Cy-3-glu induced MDA-MB-231 cell death was not through necroptosis. On the other hand, autophagy is always activated in response to metabolic stress, growth factor deprivation, limitation of nutrients and energy metabolism, or induced as an adaptive response against endoplasmic reticulum stress [45], which cannot induce the caspases cascade by TNF- $\alpha$.

In conclusion, our data revealed that Cy-3-glu directly binds to ERa36, inhibits EGFR/AKT signaling, and also promotes EGFR degradation in TNBC. Even though the Cy-3-glu concentration is high and the translational application of Cy-3-glu itself is somehow limited, our study provides an insight into the mechanism whereby the natural compounds from plants would exert potential preventive or therapeutic effects in the treatments against TNBC.

\section{MATERIALS AND METHODS}

\section{Compounds and antibodies}

Cy-3-glu was purchased from Polyphenols AS Laboratories (Hanabryggene Technology Centre, Norway). The monoclonal and polyclonal antibodies for phosphoEGFR (Tyr845) (\#6963), EGFR (\#4267), phospho-AKT (Ser473) (\#4060), cleaved-caspase-3 (\#9664), caspase-3 (\#9665), caspase-8 (\#9746) and caspase-9 (\#9508) were obtained from Cell Signaling Technology (CST, USA) and RIP3 was obtained from Abcam (ab16090, Abcam, US). The monoclonal and polyclonal antibodies for $\beta$-actin (sc-47778), AKT (sc-1619) and PCNA (sc-7907) were purchased from Santa Cruz Biotechnology, Inc. (Santa Cruz, USA). Anti-mouse or anti-rabbit secondary horseradish peroxidase (HRP) conjugate was obtained from ZSGB-BIO (Beijing, China). 17ß-estradiol (E2), Dimethylsulfoxide (DMSO), phosphate buffered saline (PBS), and other chemicals were purchased from SigmaAldrich (Sigma, USA). SNG162, an ERa36 specific inhibitor, the monoclonal antibody of ER $\alpha 36$ and ER $\alpha 36$ ligand binding domain fused with $\mathrm{Fc}$ (Fc-LBD) protein were kindly provided by Shenogen Pharma Group Inc. (Beijing, China). 


\section{Cell cultures and treatments}

The human ER and PR positive BC cell line MCF7 (ATCC ${ }^{\circledR}$ HTB-22 ${ }^{\mathrm{TM}}$ ), HER-2 positive BC cell line SK-BR-3 (ATCC $\left(\right.$ HTB-30 ${ }^{\mathrm{TM}}$ ), TNBC cell lines MDAMB-436 (ATCC $®$ HTB-130 ${ }^{\mathrm{TM}}$ ), BT-20 (ATCC $®$ HTB$19^{\mathrm{TM}}$ ) and MDA-MB-231 (ATCC ${ }^{\circ}$ CRM-HTB-26 ${ }^{\mathrm{TM}}$ ), and human non-cancerous mammary epithelial cell line (MCF-10A; ATCC ${ }^{\circledR}$ CRL-10317 ${ }^{\mathrm{TM}}$ ) were purchased from the American Type Culture Collection (Manassas, USA). MDA-MB-231-luc cell line was a gift by Dr. Bin Gao, Ph.D., Professor from Chinese Academy of Sciences. MCF-7, SK-BR-3 and BT20 cells were maintained in Dulbecco's modified Eagle's medium (DMEM; Sigma, USA) supplemented with $10 \% \mathrm{FBS}, 100 \mathrm{U} / \mathrm{mL}$ penicillin and $50 \mathrm{U} / \mathrm{mL}$ streptomycin in a humidified atmosphere containing $5 \% \mathrm{CO}_{2}$, at $37^{\circ} \mathrm{C}$. MDA-MB-231 and MDAMB-436 cells were maintained in L15 medium (Sigma, USA) supplemented with $10 \% \mathrm{FBS}, 100 \mathrm{U} / \mathrm{mL}$ penicillin and $50 \mathrm{U} / \mathrm{mL}$ streptomycin in a humidified atmosphere at $37^{\circ} \mathrm{C}$. MCF-10A cells were cultured in DMEM/Nutrient Mixture F-12 Ham medium (DMEM/F-12; Sigma, USA) containing $5 \%$ horse serum in the presence of $10 \mu \mathrm{g} / \mathrm{mL}$ insulin (Sigma, USA), $20 \mathrm{ng} / \mathrm{mL}$ epidermal growth factor (EGF; Sigma, USA), $100 \mathrm{ng} / \mathrm{mL}$ cholera toxins (Sigma, USA), $0.5 \mu \mathrm{g} / \mathrm{mL}$ hydrocortisone (Sigma, USA), $100 \mathrm{U} /$ $\mathrm{mL}$ penicillin, and $50 \mathrm{U} / \mathrm{mL}$ streptomycin. All cells were used in experiments during the linear phase of growth.

\section{Cell viability and cytotoxicity assay}

Cells were suspended at a final concentration of $4 \times$ $10^{3}$ cells/well in 96-well flat-bottomed micro plates. Cells at $60-75 \%$ confluence were treated with Cy-3-glu. After removing the culture supernatant, CCK-8 $(10 \mu \mathrm{L})$ was added to each well containing $100 \mu \mathrm{L}$ of culture medium and the plate was incubated for $2 \mathrm{~h}$ at $37^{\circ} \mathrm{C}$. Viable cells were assessed by absorbance measurements at $450 \mathrm{~nm}$ using a monochromatormicroplate reader (BioTek, USA). For cytotoxicity assay: measurement of the cell membrane integrity is a parameter for the cell death. Briefly, 2x CellTox Green Reagent (Promega, USA) included in each well at the time of Cy-3-glu dosing, and the provided lysis solution at 1:25 ratio was used as a positive control. Fluorescence was measured using a Tecan Infinite plate reader with $485-510 \mathrm{~nm}$ excitation and $520-530 \mathrm{~nm}$ emission. All experiments were performed in triplicate on three separate occasions and the data are presented as the mean of the triplicate.

\section{Cell cycle analysis}

Cells were collected and washed in cold PBS, and fixed gently in $70 \%$ ethanol overnight at $4^{\circ} \mathrm{C}$. After resuspension in PBS containing $0.01 \mathrm{mg} / \mathrm{mL}$ propidium iodide (PI; Sigma, USA) and $0.1 \mathrm{mg} / \mathrm{mL}$ RNase, cells were incubated in the dark for $30 \mathrm{~min}$ and the stained cells were analyzed with a FC-500 flow cytometer (Beckman Coulter, USA). Cell cycle distribution was analyzed using MultiCycle software (Phoenix Flow Systems, USA). For each experiment, 10,000 cells were recorded. Each experiment was run in triplicate and carried out three individual times.

\section{Apoptosis assay}

Cells were analyzed by Flow cytometer analysis. Briefly, cells treated with DMSO or Cy-3-glu were trypsinized, washed in cold PBS, dual stained with the Annexin V/PI apoptosis detection kit (Sigma, USA) following the manufacturer's instructions and signals were detected through FL-1 (FITC) and FL-3 (PI) detectors. For each analysis, 5,000-10000 cells were recorded.

\section{TUNEL staining assay}

Apoptotic cell death was confirmed by the terminal deoxynucleotidyltransferase-mediated dUTP nick endlabeling (TUNEL) technique as described by the In Situ Cell Death Detection kit, POD (Roche, Germany) for DNA chromatin morphologic features used during quantification following the manufacturer's guidelines. For quantification of apoptosis, the results were viewed under a fluorescence microscope (Olympus, Japan). At least 1000 cells from more than ten random microscopic fields were counted by two observers. We quantified TUNEL-positive staining cells by counting cells in 20 fields of each slide at an original magnification of $\times 200$ using NIH Image J 1.42 program (http://rsb.info.nih.gov/ij/ download.html). For flow cytometry: cells were fixed with $4 \%$ paraformaldehyde and then cells were resuspended in $0.1 \%$ Triton X-100 permeabilization solution, subjected to TUNEL staining, and analysed by flow cytometry using a FC-500 flow cytometer (Beckman Coulter, USA).

\section{Measurement of mitochondrial ROS}

Cells treated with DMSO or Cy-3-glu were removed from the culture medium at $24 \mathrm{~h}$ and stained with MitoTrackerRed CM-H2XRos (Invitrogen, USA) at $37^{\circ} \mathrm{C}$ in a humidified $5 \% \mathrm{CO}_{2}$ atmosphere for 30 min. Cells were then collected with trypsin, washed in cold PBS, and analyzed on a flow cytometer (Beckman Coulter, USA).

\section{SOD activity assay}

Cells treated with DMSO or Cy-3-glu for $24 \mathrm{~h}$ and cell extracts were prepared and protein concentration was determined by the Bio-Rad protein assay kit (cat. 500-0002; BioRad, USA). SOD activity was performed according to the instruction of the assay kit (Beyotime, China). All experiments were performed in triplicate in three individual times, and the data are presented as the 
mean of the triplicate. The SOD activity was expressed on $\mathrm{mg}$ protein.

\section{RNA extraction and real-time RT-PCR}

Total RNA and quantitative real-time PCR (qPCR) were performed as described previously [46]. Primer pairs are shown in Table 2. Data were analyzed with LightCycler® 480 software, Version 1.5 (Roche). Relative quantification of gene expression was performed using standard curves and normalized to the value for glyceraldehyde 3-phosphate dehydrogenase (GAPDH) in each sample. The films were scanned and quantified using the NIH Image J 1.42 program (http://rsb.info.nih.gov/ij/ download.html), each experiment was run in triplicate.

\section{Western blotting}

Protein was extracted from cell samples using the RIPA method and quantified by a Bio-Rad protein assay kit (cat. 500-0002; BioRad, USA). Protein samples were separated by SDS-PAGE and then transferred to a PVDF membrane (cat. IPVH00010; Millipore, USA). The membrane was incubated overnight at $4{ }^{\circ} \mathrm{C}$ with the primary antibody diluted in 5\% non-fat dry milk. The membranes were washed 3 times and incubated for $45 \mathrm{~min}$ at room temperature with the HRP conjugated secondary antibody (Beijing, China). Each reaction was performed in triplicate in three independent Western blotting assays. The films were scanned and quantified using the NIH Image J 1.42 program (http://rsb.info.nih.gov/ij/download.html).

\section{In vivo tumor growth and bioluminescent imaging}

Five-week-old intact female BALB/c athymic mice were purchased (Animal Center, Academy of Military Medical Sciences, China) and randomly divided into three groups ( $\mathrm{n}=10$ for each group). The control group and tumor model group were fed a normal diet (ND; $n$ = 10) (Supplementary Table S2), and the Cy-3-glu group was fed ND containing Cy-3-glu $420 \mathrm{mg} / \mathrm{kg} \mathrm{ND}(\mathrm{n}=10)$. At 6 weeks of age, mice were injected s.c. with MDAMB-231-luc cells $\left(2 \times 10^{6}\right)$ in matrigel (BD Biosciences, USA). Body weights and food intake were monitored weekly. Tumor growth was monitored in real time with bioluminescent imaging of luciferase activity in live mice using the cryogenically cooled IVIS-imaging system (Calipers, USA). Tumor size was measured weekly using calipers, and the volume was calculated with the formula $\left[\left(4 / 3 \pi r_{1}^{2} \times r_{2}\right)(0.125)\right]$, where $r_{1}$ is the smaller radius and $\mathrm{r}_{2}$ is the larger radius. At 12 weeks of age, mice were sacrificed. All animal experiments were performed in compliance with the Institutional Animal Care and Use Committee guidelines of CAU and the permission number was SKLAB-2014-01-05. Four weeks post injection, mice were euthanized and tumors were removed, weighed, and identified with hematoxylin and eosin staining and cleaved-caspase-3 histological staining.

\section{Expression and purification of LBD-ERa66}

The human LBD-ER $\alpha 66$ was expressed by PET30a vector. The inclusion bodies of bacterial pellet were resolved in buffer containing $50 \mathrm{mM}$ Tris-HCL ( $\mathrm{pH} 8.0), 100 \mathrm{mM}$ $\mathrm{NaCl}, 10 \mathrm{mM}$ EDTA, $6 \mathrm{M}$ guanidine hydrochloride, 10\% glycerin and $10 \mathrm{mM}$ DTT. After then inclusion bodies were diluted into refolding buffer (100 mM Tris [pH 8.5], 400 $\mathrm{mM}$ L-Arg $\mathrm{HCl}, 2 \mathrm{mM}$ EDTA, $5 \mathrm{mM}$ reduced glutathione and $0.5 \mathrm{mM}$ oxidized glutathione) and stirred overnight at $4^{\circ} \mathrm{C}$. Subsequently, the refolded protein was concentrated to less than $50 \mathrm{~mL}$ and exchanged into buffer containing 20 $\mathrm{mM}$ Tris-HCL (pH 8.5), $150 \mathrm{mM} \mathrm{NaCl}, 2$ mM EDTA and 2 mM DTT. Finally $2 \mathrm{~mL}$ sample was further purified by gel filtration chromatography (Superdex-20016/60 GL column, GE Healthcare).

\section{The quantification of microvasculature density}

Microvessel density (MVD) was quantified according to the methods established by Weidner [47]. Briefly, all section slides were scanned at low power $(\times 100)$ to identify the "hot spots", which represented the areas of highest neovascularization. The individual microvessels were later counted under high power $(\times 400)$ to obtain a vessel count in a defined area. The average vessel count for the five "hot spots" was calculated as the MVD. The results were calculated as endometrial MVD/ $\mathrm{mm}^{2}$ (vessels $\left./ \mathrm{mm}^{2}\right)$. Differences with $\mathrm{p}<0.01(* *), \mathrm{n}=20$ fields compared.

\section{Immunohistochemistry}

This method was performed as previously described [48]. Briefly, four micron tissue sections were deparaffinized in xylene and rehydrated through a series of decreasing ethanol concentrations. The slides were pretreated with hydrogen peroxide (3\%) for $10 \mathrm{~min}$ to remove endogenous peroxidase, followed by antigen retrieval in a microwave for $15 \mathrm{~min}$ in $10 \mathrm{mM}$ citrate buffer ( $\mathrm{pH}$ 6.0). The primary antibodies were applied, followed by washing and incubation with the biotinylated secondary antibody for $30 \mathrm{~min}$ at room temperature. The slides were counterstained with hematoxylin and dehydrated in alcohol and xylene before mounting.

\section{Microscale thermophoretic analysis}

MicroScale Thermophoresis (MST) was used to study molecular interactions in solution $[49,50]$. The binding affinity of Cy-3-glu with the purified ER $\alpha 36$ ligand binding domain (LBD) and E2 with LBD-ER $\alpha 66$ proteins were measured by MST analysis (Monolith NT. 115, NanoTemper Technologies GmbH, Germany), E2 
served as a positive control for ER $\alpha 66$ LBD binding assay. Briefly, the LBD proteins were labeled with a Cy5/Alexa 647 fluorescence dye (NT647, NanoTemper Technologies GmbH, Germany). Cy-3-glu was titrated between 0.61 and $2500 \mu \mathrm{M}$ and E2 was between 0.49 and $1000 \mathrm{nM}$ to a constant amount of purified ER $\alpha 36$ or ER $\alpha 66$ LBD proteins (100 nM). Data were analyzed using NTAnalysis software. Each reaction was performed in triplicate for three independent assays.

\section{Knocked down ERa36 in MDA-MB-231 cells}

MDA-MB-231 cells with ER $\alpha 36$ expression knocked down by the shRNA method was established previously [51]. Briefly, MDA-MB-231 cells were transfected with the empty expression vector or the ER $\alpha 36$-specific shRNA expression vector pRNAT-U6.1/Neo plasmid containing the shRNA against ER $\alpha 36$ (GenScript, USA) with Lipofectamine 3000 according to the manufacturer's instruction. Forty-eight hours after transfection, the cells were re-plated and selected with G418 (300 mg/mL) for two weeks. Clones were expanded for further analysis.

\section{Microarray data}

A list of growth-related genes (top 10) in MDAMB-231 cells compared MCF-7 cells were mined from the GEO data set GSE34987 (http://www.ncbi.nlm.nih. gov/geo/geo2r/?acc=GSE34987) [52]. Top ten candidates were obtained from the mined NCBI database with the GEO2R module.

\section{Statistical analysis}

One-way ANOVA, Dunnett's post hoc tests, Spearman and Kendall's tau-b tests were performed for statistical analyses using the SPSS18.0.1 Package (SPSS Inc., Chicago, IL). Differences with $\mathrm{p}<0.05(*), \mathrm{p}<$ $0.01(* *)$ or $\mathrm{p}<0.001(* * *)$ was considered statistically significant. Values are presented as the mean \pm SEM (standard error of mean).

\section{ACKNOWLEDGMENTS AND GRANT SUPPORT}

This work was supported by grants from the The Project for Extramural Scientists of State Key Laboratory of Agrobiotechnology (2015SKLAB6-2) to Xiangdong Li, National Science and Technology Project (2016YFD0101000; 2012CB113900) to Yang-Dong Guo, and NSFC (81372386) to Xiru Li.

\section{CONFLICTS OF INTEREST}

None.

\section{REFERENCES}

1. Reis-Filho J, Tutt A. Triple negative tumours: a critical review. Histopathology. 2008; 52: 108-18.

2. Cleator S, Heller W, Coombes RC. Triple-negative breast cancer: therapeutic options. Lancet Oncol. 2007; 8: 235-44. doi: 10.1016/S1470-2045(07)70074-8.

3. Kaufmann SH, Vaux DL. Alterations in the apoptotic machinery and their potential role in anticancer drug resistance. Oncogene. 2003; 22: 7414-30. doi: 10.1038/ sj.onc. 1206945 .

4. Changavi AA, Shashikala A, Ramji AS. Epidermal Growth Factor Receptor Expression in Triple Negative and Nontriple Negative Breast Carcinomas. J Lab Physicians. 2015; 7: 79-83. doi: 10.4103/0974-2727.163129.

5. Sobande F, Dusek L, Matejkova A, Rozkos T, Laco J, Ryska A. EGFR in triple negative breast carcinoma: significance of protein expression and high gene copy number. Cesk Patol. 2015; 51: 80-6.

6. Ueno NT, Zhang D. Targeting EGFR in Triple Negative Breast Cancer. J Cancer. 2011; 2: 324-8.

7. Hudis CA, Gianni L. Triple-negative breast cancer: an unmet medical need. Oncologist. 2011; 16: 1-11. doi: 10.1634/theoncologist.2011-S1-01.

8. Dolle JM, Daling JR, White E, Brinton LA, Doody DR, Porter PL, Malone KE. Risk factors for triple-negative breast cancer in women under the age of 45 years. Cancer Epidemiol Biomarkers Prev. 2009; 18: 1157-66. doi: 10.1158/1055-9965.EPI-08-1005.

9. Zhang XT, Kang LG, Ding L, Vranic S, Gatalica Z, Wang ZY. A positive feedback loop of ER-alpha36/ EGFR promotes malignant growth of ER-negative breast cancer cells. Oncogene. 2011; 30: 770-80. doi: 10.1038/ onc. 2010.458 .

10. Wang Z, Zhang X, Shen P, Loggie BW, Chang Y, Deuel TF. A variant of estrogen receptor- $\{$ alpha $\}$, hER\{alpha\}36: transduction of estrogen- and antiestrogendependent membrane-initiated mitogenic signaling. Proc Natl Acad Sci U S A. 2006; 103: 9063-8. doi: 10.1073/ pnas.0603339103.

11. Brand TM, Iida M, Dunn EF, Luthar N, Kostopoulos KT, Corrigan KL, Wleklinski MJ, Yang D, Wisinski KB, Salgia R, Wheeler DL. Nuclear Epidermal Growth Factor Receptor Is a Functional Molecular Target in Triple-Negative Breast Cancer. Molecular Cancer Therapeutics. 2014; 13: 1356-68. doi: 10.1158/1535-7163.MCT-13-1021.

12. Wu X, Beecher GR, Holden JM, Haytowitz DB, Gebhardt SE, Prior RL. Concentrations of anthocyanins in common foods in the United States and estimation of normal consumption. J Agric Food Chem. 2006; 54: 4069-75. doi: 10.1021/jf0603001.

13. KR AOaM. Flavonoids: chemistry, biochemistry and applications. CRC Press. 2010. 
14. Song J, Zhao MY, Liu X, Zhu YC, Hu XS, Chen F. Protection of cyanidin-3-glucoside against oxidative stress induced by acrylamide in human MDA-MB-231 cells. Food and Chemical Toxicology. 2013; 58: 306-10. doi: 10.1016/j. fct.2013.05.003.

15. Min S-W, Ryu S-N, Kim D-H. Anti-inflammatory effects of black rice, cyanidin-3-O- $\beta$-D-glycoside, and its metabolites, cyanidin and protocatechuic acid. International immunopharmacology. 2010; 10: 959-66.

16. Song NR, Yang H, Park J, Kwon JY, Kang NJ, Heo YS, Lee KW, Lee HJ. Cyanidin suppresses neoplastic cell transformation by directly targeting phosphatidylinositol 3-kinase. Food Chemistry. 2012; 133: 658-64.

17. Chen P-N, Chu S-C, Chiou H-L, Chiang C-L, Yang S-F, Hsieh Y-S. Cyanidin 3-glucoside and peonidin 3-glucoside inhibit tumor cell growth and induce apoptosis in vitro and suppress tumor growth in vivo. Nutrition and cancer. 2005; 53: 232-43.

18. Kong JM, Chia LS, Goh NK, Chia TF, Brouillard R. Analysis and biological activities of anthocyanins. Phytochemistry. 2003; 64: 923-33.

19. Welch CR, Wu Q, Simon JE. Recent Advances in Anthocyanin Analysis and Characterization. Curr Anal Chem. 2008; 4: 75-101. doi: 10.2174/157341108784587795.

20. Kerr JF, Wyllie AH, Currie AR. Apoptosis: a basic biological phenomenon with wide-ranging implications in tissue kinetics. British journal of cancer. 1972; 26: 239 .

21. Festjens N, Vanden Berghe T, Vandenabeele P. Necrosis, a well-orchestrated form of cell demise: signalling cascades, important mediators and concomitant immune response. Biochimica et Biophysica Acta (BBA)-Bioenergetics. 2006; 1757: 1371-87.

22. Ashkenazi A, Dixit VM. Apoptosis control by death and decoy receptors. Current opinion in cell biology. 1999; 11: 255-60.

23. McCord JM, Keele BB, Jr., Fridovich I. An enzyme-based theory of obligate anaerobiosis: the physiological function of superoxide dismutase. Proc Natl Acad Sci U S A. 1971; 68: 1024-7.

24. Logue SE, Martin SJ. Caspase activation cascades in apoptosis. Biochem Soc Trans. 2008; 36: 1-9. doi: 10.1042/ BST0360001.

25. Cui M, Zhang Y, Liu S, Xie W, Ji M, Lou H, Li X. 1-oxoeudesm-11(13)-ene-12,8alpha-lactone-induced apoptosis via ROS generation and mitochondria activation in MCF-7 cells. Arch Pharm Res. 2011; 34: 1323-9. doi: 10.1007/ s12272-011-0812-x.

26. Huttemann M, Pecina P, Rainbolt M, Sanderson TH, Kagan VE, Samavati L, Doan JW, Lee I. The multiple functions of cytochrome $\mathrm{c}$ and their regulation in life and death decisions of the mammalian cell: From respiration to apoptosis. Mitochondrion. 2011; 11: 369-81. doi: 10.1016/j. mito.2011.01.010.
27. Tsai EM, Wang SC, Lee JN, Hung MC. Akt activation by estrogen in estrogen receptor-negative breast cancer cells. Cancer Res. 2001; 61: 8390-2.

28. Wienken CJ, Baaske P, Rothbauer U, Braun D, Duhr S. Protein-binding assays in biological liquids using microscale thermophoresis. Nat Commun. 2010; 1: 100. doi: $10.1038 /$ ncomms 1093 .

29. Yarden Y, Sliwkowski MX. Untangling the ErbB signalling network. Nature reviews Molecular cell biology. 2001; 2: 127-37.

30. Orlowski RZ. The role of the ubiquitin-proteasome pathway in apoptosis. Cell Death Differ. 1999; 6: 303-13. doi: 10.1038/sj.cdd.4400505.

31. Tanaka K, Chiba T. The proteasome: a protein-destroying machine. Genes Cells. 1998; 3: 499-510.

32. Hollestelle A, Nagel JHA, Smid M, Lam S, Elstrodt F, Wasielewski M, Ng SS, French PJ, Peeters JK, Rozendaal MJ, Riaz M, Koopman DG, ten Hagen TLM, et al. Distinct gene mutation profiles among luminal-type and basal-type breast cancer cell lines. Breast Cancer Research and Treatment. 2010; 121: 53-64. doi: 10.1007/ s10549-009-0460-8.

33. Adams LS, Phung S, Yee N, Seeram NP, Li L, Chen S. Blueberry phytochemicals inhibit growth and metastatic potential of MDA-MB-231 breast cancer cells through modulation of the phosphatidylinositol 3-kinase pathway. Cancer research. 2010; 70: 3594-605.

34. Adams LS, Kanaya N, Phung S, Liu Z, Chen S. Whole blueberry powder modulates the growth and metastasis of MDA-MB-231 triple negative breast tumors in nude mice. The Journal of nutrition. 2011; 141: 1805-12.

35. Wang ZY, Zhang XT, Shen P, Loggie BW, Chang YC, Deuel TF. Identification, cloning, and expression of human estrogen receptor-alpha 36, a novel variant of human estrogen receptor-alpha 66. Biochemical and Biophysical Research Communications. 2005; 336: 1023-7. doi: 10.1016/j.bbrc.2005.08.226.

36. Lin SL, Yan LY, Zhang XT, Yuan J, Li M, Qiao J, Wang ZY, Sun QY. ER-alpha36, a variant of ER-alpha, promotes tamoxifen agonist action in endometrial cancer cells via the MAPK/ERK and PI3K/Akt pathways. PLoS One. 2010; 5: e9013. doi: 10.1371/journal.pone.0009013.

37. Kang L, Zhang X, Xie Y, Tu Y, Wang D, Liu Z, Wang ZY. Involvement of estrogen receptor variant ER-alpha36, not GPR30, in nongenomic estrogen signaling. Mol Endocrinol. 2010; 24: 709-21. doi: 10.1210/me.2009-0317.

38. Kang L, Guo Y, Zhang X, Meng J, Wang ZY. A positive cross-regulation of HER2 and ER-alpha36 controls ALDH1 positive breast cancer cells. J Steroid Biochem Mol Biol. 2011; 127: 262-8. doi: 10.1016/j. jsbmb.2011.08.011.

39. Su Z, Yang Z, Xu Y, Chen Y, Yu Q. Apoptosis, autophagy, necroptosis, and cancer metastasis. Mol Cancer. 2015; 14: 48. doi: 10.1186/s12943-015-0321-5. 
40. Jung S, Li C, Duan J, Lee S, Kim K, Park Y, Yang Y, Kim KI, Lim JS, Cheon CI, Kang YS, Lee MS. TRIP-Br1 oncoprotein inhibits autophagy, apoptosis, and necroptosis under nutrient/serum-deprived condition. Oncotarget. 2015; 6: 29060-75. doi: 10.18632/oncotarget.5072 5072.

41. Lee EW, Kim JH, Ahn YH, Seo J, Ko A, Jeong M, Kim SJ, Ro JY, Park KM, Lee HW, Park EJ, Chun KH, Song J. Ubiquitination and degradation of the FADD adaptor protein regulate death receptor-mediated apoptosis and necroptosis. Nat Commun. 2012; 3: 978. doi: 10.1038/ ncomms 1981.

42. Sun W, Bao JL, Lin W, Gao HW, Zhao WW, Zhang QW, Leung CH, Ma DL, Lu JJ, Chen XP. 2-Methoxy-6-acetyl7-methyljuglone (MAM), a natural naphthoquinone, induces NO-dependent apoptosis and necroptosis by $\mathrm{H} 2 \mathrm{O} 2-$ dependent JNK activation in cancer cells. Free Radical Biology and Medicine. 2016; 92: 61-77. doi: 10.1016/j. freeradbiomed.2016.01.014.

43. Sun L, Wang H, Wang Z, He S, Chen S, Liao D, Wang L, Yan J, Liu W, Lei X. Mixed lineage kinase domain-like protein mediates necrosis signaling downstream of RIP3 kinase. Cell. 2012; 148: 213-27.

44. He S, Wang L, Miao L, Wang T, Du F, Zhao L, Wang X. Receptor interacting protein kinase-3 determines cellular necrotic response to TNF- $\alpha$. Cell. 2009; 137: 1100-11.

45. Nikoletopoulou V, Markaki M, Palikaras K, Tavernarakis N. Crosstalk between apoptosis, necrosis and autophagy. Biochimica et Biophysica Acta (BBA)-Molecular Cell Research. 2013.

46. Yu W, Zheng H, Lin W, Tajima A, Zhang Y, Zhang X, Zhang H, Wu J, Han D, Rahman NA, Korach KS, Gao GF, Inoue I, et al. Estrogen promotes Leydig cell engulfment by macrophages in male infertility. J Clin Invest. 2014; 124: 2709-21. doi: 10.1172/JCI59901 59901 [pii].

47. Weidner N. Current pathologic methods for measuring intratumoral microvessel density within breast carcinoma and other solid tumors. Breast Cancer Res Treat. 1995; 36: 169-80

48. Li X, Strauss L, Makela S, Streng T, Huhtaniemi I, Santti R, Poutanen M. Multiple structural and functional abnormalities in the p450 aromatase expressing transgenic male mice are ameliorated by a $\mathrm{p} 450$ aromatase inhibitor. Am J Pathol. 2004; 164: 1039-48. doi: 10.1016/ S0002-9440(10)63191-4.

49. Shang X, Marchioni F, Evelyn CR, Sipes N, Zhou X, Seibel W, Wortman M, Zheng Y. Small-molecule inhibitors targeting G-protein-coupled Rho guanine nucleotide exchange factors. Proc Natl Acad Sci U S A. 2013; 110: 3155-60. doi: 10.1073/pnas.1212324110.

50. Bayer H, Essig K, Stanzel S, Frank M, Gildersleeve JC, Berger MR, Voss C. Evaluation of riproximin binding properties reveals a novel mechanism for cellular targeting. J Biol Chem. 2012; 287: 35873-86. doi: 10.1074/jbc. M112.368548.

51. Kang L, Wang ZY. Breast cancer cell growth inhibition by phenethyl isothiocyanate is associated with down-regulation of oestrogen receptor-alpha36. J Cell Mol Med. 2010; 14: 1485-93. doi: 10.1111/j.1582-4934.2009.00877.x.

52. Nguyen-Vu T, Vedin LL, Liu K, Jonsson P, Lin JZ, Candelaria NR, Candelaria LP, Addanki S, Williams C, Gustafsson JA, Steffensen KR, Lin CY. Liver x receptor ligands disrupt breast cancer cell proliferation through an E2F-mediated mechanism. Breast Cancer Research. 2013; 15. doi: Artn R51 10.1186/Bcr3443. 